\title{
EXISTENCE AND UNIQUENESS \\ OF THE RIEMANN PROBLEM FOR A NONLINEAR SYSTEM OF CONSERVATION LAWS OF MIXED TYPE
}

\author{
L. HSIAO AND P. DE MOTTONI
}

ABSTRACT. We study the system of conservation laws given by

$$
\left\{\begin{array}{l}
u_{t}+[u(1-v)]_{x}=0, \\
v_{t}+[v(a+u)]_{x}=0 \quad(a>1 \text { is a constant }),
\end{array}\right.
$$

with any Riemann initial data $\left(u_{\mp}, v_{\mp}\right)$.

The system is elliptic in the domain where $(v-u+a-1)^{2}+4(a-1) u<0$ and strictly hyperbolic when $(v-u+a-1)^{2}+4(a-1) u>0$. We combine and generalize Lax criterion and Oleinik-Liu criterion to introduce the generalized entropy condition (G.E.C.) by which we can show that the Riemann problem always has a weak solution (any discontinuity satisfies the G.E.C.) for any initial data, however not necessarily unique. We introduce the minimum principle then in the definition of an admissible weak solution for the Riemann problem and the existence and uniqueness of the solution for any Riemann data.

\section{INTRODUCTION}

Suppose $U(x, t), V(x, t)$ describe the densities of two biological populations, the second pursuing the first along a straight line course, in such a way that the $U$-population provokes the $V$-population into the pursuing action by moving away, and the $V$-population causes the $U$-population to escape by running after it. More specifically, following Murray and Cohen [MC], let us assume that the $U$ 's escape from the $V$ 's at a rate proportional to the space gradient of the $V$ 's and, in turn, the $V$ 's try to approach the $U$ 's at a rate proportional to the space gradient of the $U$ 's. Since the equation of motion can be written as

$$
U_{t}+\lambda_{1} U_{x}=0, \quad V_{t}+\lambda_{2} V_{x}=0,
$$

$\lambda_{1}, \lambda_{2}$ representing the advection velocities of the $U$ 's, respectively $V$ 's, we obtain Murray and Cohen's equations (without zeroth-order interaction):

$$
U_{t}+\left(\alpha-\beta V_{x}\right) U_{x}=0, \quad V_{t}+\left(\gamma+\delta U_{x}\right) V_{x}=0
$$

for some positive constants $\alpha, \beta, \gamma, \delta$. Redefining $U \rightarrow U / \delta, V \rightarrow V / \beta$, and rescaling the space and time respectively, we arrive at the normalized form

$$
U_{t}+\left(1-V_{x}\right) U_{x}=0, \quad V_{t}+\left(a+U_{x}\right) V_{x}=0
$$

where $a=\gamma / \alpha$.

Received by the editors November 4, 1987 and, in revised form, May 6, 1988.

1980 Mathematics Subject Classification (1985 Revision). Primary 35L67, 35L80, 35L45; Secondary $92 \mathrm{~A} 15,92 \mathrm{~A} 17,92 \mathrm{~A} 90$. 
Putting $U_{x}=u, V_{x}=v$, we obtain the following system of conservation laws:

$$
\left\{\begin{array}{l}
u_{t}+[u(t-v)]_{x}=0 \\
v_{t}+[v(a+u)]_{x}=0
\end{array}\right.
$$

For the case $a=1$, which means that in the absence of interaction, the two populations run with the same velocity, -1 , the system becomes into

$$
\left\{\begin{array}{l}
u_{t}+[u(1-v)]_{x}=0 \\
v_{t}+[v(1+u)]_{x}=0
\end{array}\right.
$$

which is not strictly hyperbolic, since its characteristic speeds coincide at $u=$ $v$. Moreover, for each $i \quad(i=1,2)$, the $i$ th characteristic field is genuinely nonlinear in one half of the phase-plane, $(u<v$ for $i=1, u>v$ for $i=2)$ and linearly degenerate in another half. Another feature of the system (1.2) is that shock and rarefaction curves coincide. (Other different systems sharing this property have been considered in the literature, in connection with various models in applied sciences, see the paper by Temple [T]. However, our model does not satisfy their assumption.) Such a feature in our model simplifies the structure of the solutions of the Riemann problem and of the wave interactions, and makes it possible to solve the Cauchy problem for arbitrary data of bounded variation $[\mathrm{HM}]$.

However, the case $a \neq 1$ leads to a different, much more involved, mathematical situation which provides an interesting example of one class of equation that may change type from hyperbolic to elliptic as the state variable varies on the phase plane. It is an open problem to determine the extent to which the Cauchy problem is meaningful for such systems. For a first step, let us study the simplest Cauchy problem-Riemann problem, namely,

$$
\left.(u, v)\right|_{t=0}= \begin{cases}\left(u_{-}, v_{-}\right), & x<0, \\ \left(u_{+}, v_{+}\right), & x>0,\end{cases}
$$

where $\left(u_{\mp}, v_{\mp}\right)$ are arbitrary constant states.

As a simplest model of mixed type system, the following quasilinear system

$$
\left\{\begin{array}{l}
u_{t}-\sigma(v)_{x}=0 \\
v_{t}-u_{x}=0
\end{array}\right.
$$

with $\sigma$ given by a nonmonotone function has been discussed by a number of people. The elliptic domain of (1.4) takes very simple form in the phase plane which is a strip $v_{\alpha}<v<v_{\beta}$, for instance, in the equations governing isothermal motion of a Van der Waals fluid. The Riemann problem of (1.4), (1.3) has been solved [Ha, $\left.\mathrm{Hs}_{1}, \mathrm{~J}, \mathrm{~K}, \mathrm{Se}, \mathrm{Sl}\right]$.

A similar model to (1.1) on two-direction traffic flow was discussed by Bick and Newell [BN]. However, they did not solve the Riemann problem for states in the elliptic region which is ellipsoidal. 
A recent paper by Holden [Ho] dealt with a quadratic system (1.5) also which is elliptic inside a circle and strictly hyperbolic

$$
\left(\begin{array}{l}
u \\
v
\end{array}\right)_{t}+\left(\begin{array}{c}
v^{2}-u^{2}+2 \rho v \\
2 u v-2 \rho u
\end{array}\right)_{x}=0 \quad(\rho \text { is a positive constant })
$$

outside it and the characteristic speed is constant on the boundary of the elliptic region. As $\rho \rightarrow 0,(1.5)$ approaches the model discussed in [SS] which is strictly hyperbolic except at origin where the eigenvalues are degenerate.

For our model (1.1), the elliptic region is unbounded on the boundary of which characteristic speed is not constant. Moreover, model (1.5) has more symmetry than (1.1) and the structure of the fognals, i.e. the curves where genuine nonlinearity fails, is different. Furthermore, the Hugoniot locus exhibits different structure. It may consist of at most three detached branches for (1.5) which may have a loop starting and ending at $\left(u_{0}, v_{0}\right)$. For our model, the Hugoniot locus can be at most three disconnected branches for each family which is true even when $\left(u_{0}, v_{0}\right)$ is in the strictly hyperbolic region. Moreover, the Hugoniot locus can be parametrized by $u$ for the first family and by $v$ for the second family.

By virtue of the geometry in (1.5), it is possible for the author to follow Liu's construction [Liu] to solve the Riemann problem though certain regions exhibit multiple solutions. It is impossible for us, however, to solve the problem in the same way. We have to combine and generalize Lax criterion and Liu-Oleinik criterion to introduce our generalized entropy condition, G.E.C. by which we can show that the Riemann problem always has a weak solution in which any discontinuity satisfies the G.E.C. for any initial data. Referring to the Lax criterion, a shock satisfying our G.E.C. can be undercompressive but not overcompressive. There is a complete classification in $\S 2$, for proof see $\left[\mathrm{Hs}_{2}\right]$. However, the same as in [Ho], certain regions exhibit multiple solutions. In order to have uniqueness we proposed a minimum principle in the definition of an admissible weak solution which is based on mathematical consideration, particularly, the continuous dependence principle. The entire $U=(u, v)$ plane is divided into a few regions, the topological structure of admissible wave curves keep the same as $U_{-}=\left(u_{-}, v_{-}\right)$varies in each one of the regions. The topology may vary only when $U_{-}$across the boundaries in the $U_{-}$-plane. In other words, for fixed $U_{-}=\left(u_{-}, v_{-}\right)$, the admissible weak solution of the Riemann problem may divide the $U_{+}$-plane $\left(U_{+}=\left(u_{+}, v_{+}\right)\right)$into as many as 14 regions, representing different combinations of shocks, rarefaction waves and composite waves, the qualitative structure of the $U_{+}$-regions changes only as $U_{-}$cross the $U_{-}$-boundary curves in the $U_{-}$-plane. (See $\left[\mathrm{HS}_{2}\right]$ for details.)

We concentrate on existence and uniqueness in this paper. In $\S 2$ we give a detailed description of the basic ingredients in the solution of the Riemann problem-the Hugoniot loci and the rarefaction wave curves first-and introduce the generalized entropy condition G.E.C. then with which a complete description of shock wave sets is given. The comparison of the G.E.C. to Lax 
criterion is discussed finally. In $\S 3$ we introduce the minimum principle in the definition of an admissible weak solution for the Riemann problem and prove the existence and uniqueness of the solution. For detailed discussion about the structure and qualitative behavior of the solution, see [ $\left.\mathrm{Hs}_{2}\right]$.

The approach introduced in this paper can be used, it seems, for a class of more general systems of mixed type for which the Hugoniot loci can be parametrized and which is "predominately hyperbolic", namely, the elliptic domain has the following property: there is at least one direction on the $(u, v)$ plane such that for any given straight line, parallel to this direction, the intersection of the elliptic domain with the straight line is finite in length.

\section{Preliminaries}

To explain the above conclusion, we list certain basic facts about (1.1) first. The $i$-characteristic speed $\lambda_{i} \quad(i=1,2)$ of $(1.1)$ is defined by

$$
\left\{\begin{array}{l}
\lambda_{1}=\frac{1}{2}\left\{u-v+a+1-\left[(v-u+a-1)^{2}+4(a-1) u\right]^{1 / 2}\right\}, \\
\lambda_{2}=\frac{1}{2}\left\{u-v+a+1+\left[(v-u+a-1)^{2}+4(a-1) u\right]^{1 / 2}\right\} .
\end{array}\right.
$$

at any $(u, v)$ where $(v-u+a-1)^{2}+4(a-1) u \geq 0$. The elliptic domain where $(v-u+a-1)^{2}+4(a-1) u<0$ is shown in Figure 2.1 where the quantity $(v-u+a-1)^{2}+4(a-1) u=(u-v+a-1)^{2}+4(a-1) v$ is denoted by $\Delta(u, v)$.

The right eigenvector $r_{i}$ corresponding to $\lambda_{i}$ can be chosen as

$$
\left\{\begin{array}{l}
r_{i}=\left(-u, \lambda_{i}-1+v\right)^{T} \text { or } \\
r_{i}=\left(\lambda_{i}-a-u, v\right)^{T}
\end{array}\right.
$$

which defines the $i$-rarefaction wave curve (see Figure 2.2), $i=1,2$, denoted by $R_{1}$ and $R_{2}$. Recall that $i$-rarefaction wave for $(1.1)(i=1,2)$ is a smooth single-valued, similarity solution $u=u(\xi), v=v(\xi)$, such that the $i$ th characteristic speed is increasing in $\xi$.

The solid lines in Figure 2.2 denote $R_{1}$ while dotted lines denote $R_{2}$; on either family of lines, $\lambda_{i}$ increases along the displayed direction. The curves

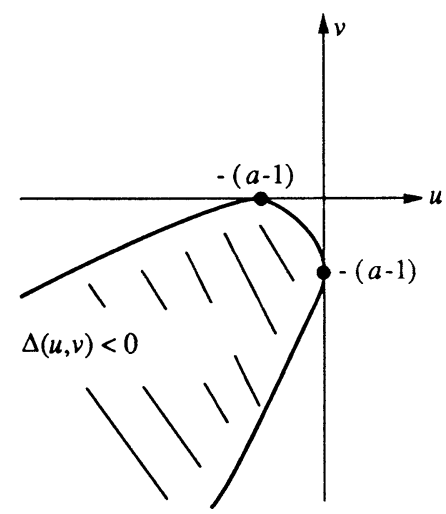

Figure 2.1 


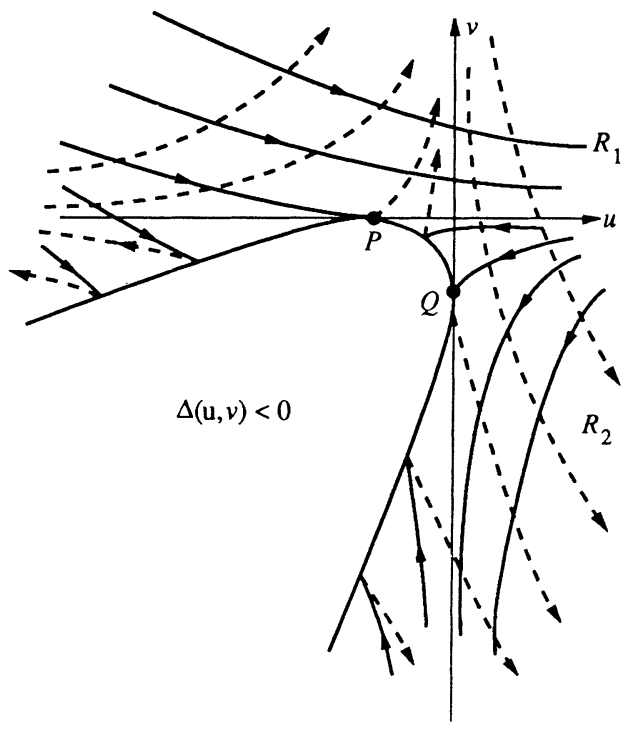

FigURE 2.2

$u=0$ and $v=0$ supply the set where genuine nonlinearity fails. More precisely, $\lambda_{1}=1$ on $v=0, u \geq-(a-1)$ and $\lambda_{1}=a$ on $u=0, v \leq-(a-1)$; $\lambda_{2}=1$ on $v=0, u \leq-(a-1)$ and $\lambda_{2}=a$ on $u=0, u \geq-(a-1)$.

A discontinuity is characterized by the left, respectively, right-hand value at the discontinuity, and by its speed, such quantities are mutually related by the well-known Rankine-Hugoniot condition, namely

$$
\left\{\begin{array}{l}
\sigma[u]=[(1-v) u] \\
\sigma[v]=[(a+u) v]
\end{array}\right.
$$

where $[w]=w_{r}-w_{l}$ denotes the jump of the quantity $w$ across the discontinuity and $\sigma$ denotes the discontinuity's speed.

For any given $\left(u_{0}, v_{0}\right)$, the set of all states $(u, v)$ which can be joined to $\left(u_{0}, v_{0}\right)$ by a discontinuity satisfying $(2.3)$ is called Hugoniot locus. It turns out that there are two types of Hugoniot loci, defined by (2.3), denoted by $H_{1}$, $\mathrm{H}_{2}$.

Indeed, (2.3) has two solutions $\left(\sigma_{1}, H_{1}\right),\left(\sigma_{2}, H_{2}\right)$, where $\sigma_{i} \in \mathbb{R}_{1}, H_{i} \subset$ $\mathbb{R} \times \mathbb{R}(i=1,2)$. They are characterized (implicitly) as follows

$$
\begin{gathered}
\sigma_{1}=\frac{1}{2}\left\{u-v_{0}+a+1-\left[\left(v_{0}-u+a-1\right)^{2}+4(a-1) u\right]^{1 / 2}\right\}, \\
\left(\sigma_{1}-a-u\right)\left(v-v_{0}\right)-v_{0}\left(u-u_{0}\right)=0, \\
H_{1}=\{(u, v):(u, v) \text { satisfying }(2.4 .1),(2.5 .1)\}
\end{gathered}
$$

and

$$
\begin{gathered}
\sigma_{2}=\frac{1}{2}\left\{u_{0}-v+a+1+\left[\left(v-u_{0}+a-1\right)^{2}+4(a-1) u_{0}\right]^{1 / 2}\right\} \\
\left(\sigma_{2}-1+v\right)\left(u-u_{0}\right)+u_{0}\left(v-v_{0}\right)=0 \\
H_{2}=\{(u, v):(u, v) \text { satisfying }(2.4 .2),(2.5 .2)\}
\end{gathered}
$$


Eliminating $\sigma_{1}$ from $(2.4 .1),(2.5 .1)$, whenever well defined, provides $H_{1}$ as the graph of a (single-valued) function of $u$; accordingly, $\sigma_{1}$ is expressed through (2.4.1) as a (single-valued) function of $u$, too:

$$
\sigma_{1}=\sigma_{1}\left(u ; u_{0}, v_{0}\right) \text {. }
$$

Likewise, eliminating $\sigma_{2}$ from (2.4.2), (2.5.2) (whenever well defined) provides $\mathrm{H}_{2}$ as the graph of a (single-valued) function of $v, \sigma_{2}$ being expressed through (2.4.2) as a (single-valued) function of $v$;

$$
\sigma_{2}=\sigma_{2}\left(v ; u_{0}, v_{0}\right)
$$

Alternatively, $\left(\sigma_{i}, H_{i}\right)$ could be characterized equivalently as follows:

$$
\begin{gathered}
\sigma=\frac{1}{2}\left\{u_{0}-v+a+1-\left[\left(v-u_{0}+a-1\right)^{2}+4(a-1) u_{0}\right]^{1 / 2}\right\} \\
\left(\sigma_{1}-1+v\right)\left(u-u_{0}\right)+u_{0}\left(v-v_{0}\right)=0
\end{gathered}
$$

and

$$
\begin{gathered}
\sigma_{2}=\frac{1}{2}\left\{u-v_{0}+a+1+\left[\left(v_{0}-u+a-1\right)^{2}+4(a-1) u\right]^{1 / 2}\right\}, \\
\left(\sigma_{2}-a-u\right)\left(v-v_{0}\right)-v_{0}\left(u-u_{0}\right)=0 .
\end{gathered}
$$

Again, eliminating $\sigma_{1}$ from (2.6.1), (2.7.1) (whenever well defined) provides $H_{1}$ as the graph of a function of $v$, and $\sigma_{1}$ being a function of $v$. Similarly to $\sigma_{2}$ and $H_{2}$ from (2.6.2) (2.7.2) with $u$ instead of $v$.

Note that the $H_{i}$ 's need not be, in general, connected curves. It will be shown that the Hugoniot locus $H_{i}$ can be at most three disconnected branches for $i=1$ or $i=2$ which is true even when $\left(u_{0}, v_{0}\right)$ is in the strictly hyperbolic region. The explicit construction of $H_{i}$ as the graph of a single-valued function requires a distinction of several cases, according to the location of the state $\left(u_{0}, v_{0}\right)$. This will be done in the following.

Case 1. $u_{0}>0, v_{0}>0$.

Due to $a>1$ and $v_{0}>0$, the function $\left(v_{0}-u+a-1\right)^{2}+4(a-1) u$ is positive for any $u \in(-\infty, \infty)$, therefore (2.4.1) supplies $\sigma_{1}$. Substituting this $\sigma_{1}$ into (2.5.1), one obtains $H_{1}$ as the graph of a function of $u$, namely:

$$
v=v_{0} \cdot \frac{u-2 u_{0}-v_{0}+1-a-\sqrt{\left(v_{0}-u+a-1\right)^{2}+4(a-1) u}}{-\left(u+v_{0}+a-1\right)-\sqrt{\left(v_{0}-u+a-1\right)^{2}+4(a-1) u}} .
$$

It is easy to check that the right-hand side is positive for any value of $u$ and $v \rightarrow 0$ as $u \rightarrow+\infty$ while $v \rightarrow+\infty$ as $u \rightarrow-\infty$, as shown in Figure 2.3.

On the other hand, $\mathrm{H}_{2}$ can be defined as the graph of a function of $v$ using (2.4.2) and (2.5.2):

$$
u=u_{0} \cdot \frac{u_{0}-v+2 v_{0}+a-1+\sqrt{\left(v-u_{0}+a-1\right)^{2}+4(a-1) u_{0}}}{u_{0}+v+a-1+\sqrt{\left(v-u_{0}+a-1\right)^{2}+4(a-1) u_{0}}}
$$




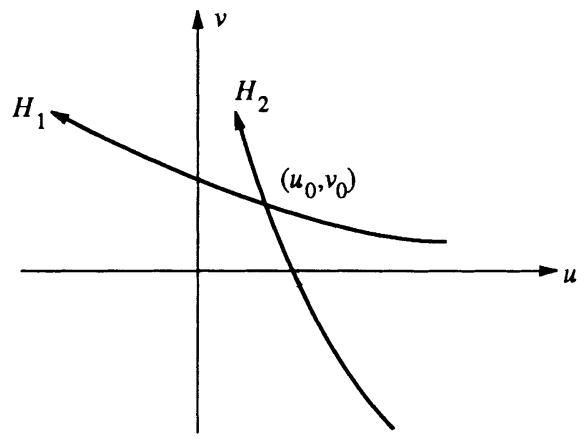

FIGURE 2.3

The right-hand side is positive for any value of $v$ and $u \rightarrow 0$ as $v \rightarrow+\infty$ while $u \rightarrow+\infty$ as $v \rightarrow-\infty$.

According to the general scheme laid down above, observe that $H_{1}$ can be defined as a function of $v$ also by using (2.7.1) and (2.6.1) (only for $v>0$ ). The same is true for $\mathrm{H}_{2}$ with $u$ (only for $u>0$ ).

Remark 2.1. When $v_{0} \rightarrow 0$ or $u_{0} \rightarrow 0$, the distribution of the Hugoniot loci changes continuously into the graph shown in Figure $2.3_{v_{0}}$ or Figure $2.3_{u_{0}}$ respectively. This holds where the ray $v=0, u \geq-(a-1)$ belongs to $H_{1}$ in Figure $2.3_{v_{0}}$ while the ray $u=0, v \geq-(a-1)$ belongs to $H_{2}$ in Figure $2.3_{u_{0}}$.

Case 2. $u_{0}>0, v_{0}<0$.

There are two subcases: $v_{0}>-(a-1)$ or $v_{0}<-(a-1)$.

$$
0>v_{0}>-(a-1) \text {. }
$$

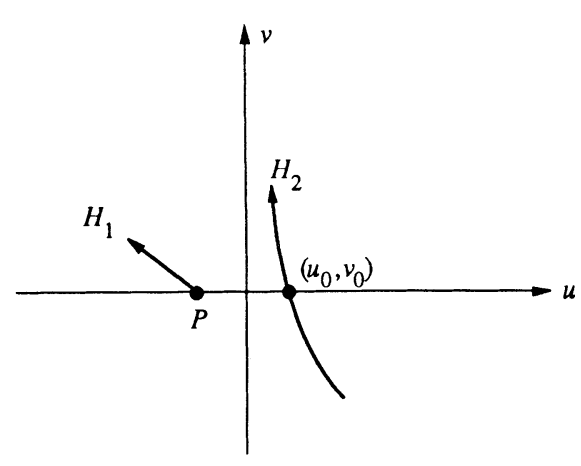

FIGURE $2.3_{v_{0}}$

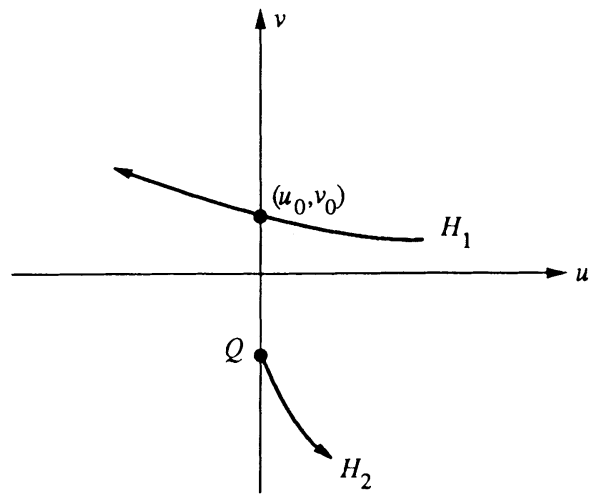

FIGURE $2.3_{u_{0}}$ 
Since $v_{0}<0$, it turns out that $\left(v_{0}-u+a-1\right)^{2}+4(a-1) u=0$ has two real roots, denoted by $u^{(m)}\left(v_{0}\right)$ and $u^{(M)}\left(v_{0}\right)$ respectively, namely

$$
\begin{aligned}
& u^{(m)}\left(v_{0}\right)=-\left(\sqrt{a-1}+\sqrt{-v_{0}}\right)^{2}, \\
& u^{(M)}\left(v_{0}\right)=-\left(\sqrt{a-1}-\sqrt{-v_{0}}\right)^{2} .
\end{aligned}
$$

For $u>u^{(M)}\left(v_{0}\right), H_{1}$ can be defined by (2.8), this provides an arc with end-point $\widetilde{C} \equiv\left(u^{(M)}\left(v_{0}\right), v_{\widetilde{C}}\right)$ where

$$
v_{\widetilde{C}}=\frac{-\sqrt{-v_{0}}}{\sqrt{a-1}-\sqrt{-v_{0}}} u_{0}-\sqrt{(a-1)\left(-v_{0}\right)} .
$$

At this point $\tilde{C}, H_{1}$ is tangent to the line $u=u^{(M)}\left(v_{0}\right)$. For $u<u^{(m)}\left(v_{0}\right)$, $H_{1}$ can be defined by (2.8) again: it is an arc originating at the point $\underset{\sim}{C} \equiv$ $\left(u^{(m)}\left(v_{0}\right), v_{C}\right)$ where

$$
v_{C}=\frac{\sqrt{-v_{0}}}{\sqrt{a-1}+\sqrt{-v_{0}}} u_{0}+\sqrt{(a-1)\left(-v_{0}\right)}
$$

and which is tangent at $\underset{\sim}{C}$ to the line $u=u^{(m)}\left(v_{0}\right)$ (see Figure 2.4). Moreover, along the curve $H_{1}, v \rightarrow 0^{-}$as $u \rightarrow+\infty$ while $v \rightarrow+\infty$ as $u \rightarrow-\infty$.

As for $H_{2},(2.9)$ shows that $H_{2}$ can be defined for any $v \in(-\infty, \infty)$ similarly to Case 1 (see Figure 2.4).

Remark 2.2. It is easy to see from the formulae $(2.10)-(2.13)$ that the points $\widetilde{C}$ and $\underset{\sim}{C}$ tend to the same point $P=(-(a-1), 0)$ as $v_{0} \rightarrow 0$. This, combined with Remark 2.1, implies that the distribution of the Hugoniot loci varies continuously when $\left(u_{0}, v_{0}\right)$ across the ray: $v=0, u \geq 0$. On the other

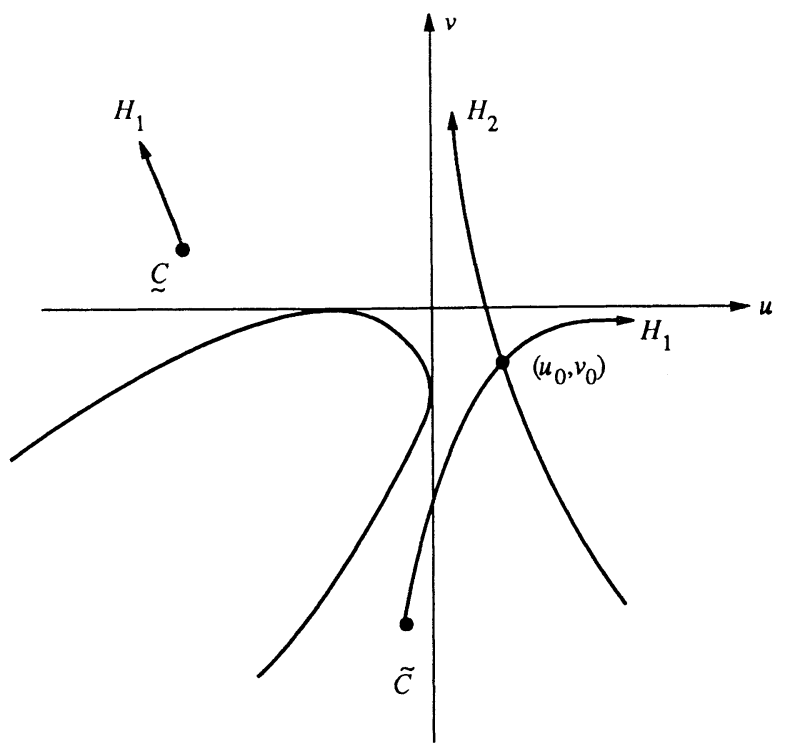

FIGURE 2.4 


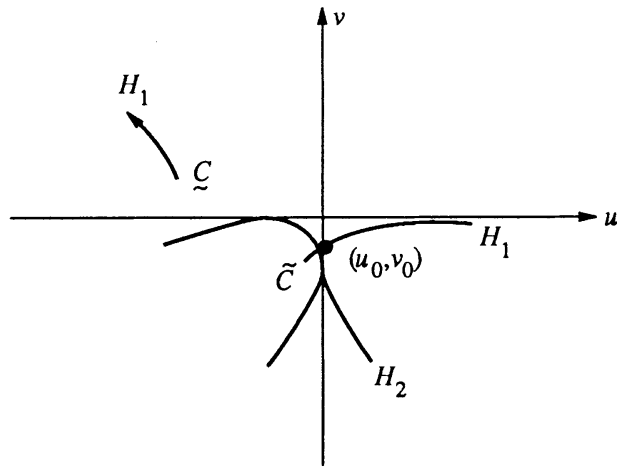

FIGURE $2.4_{u_{0}}$

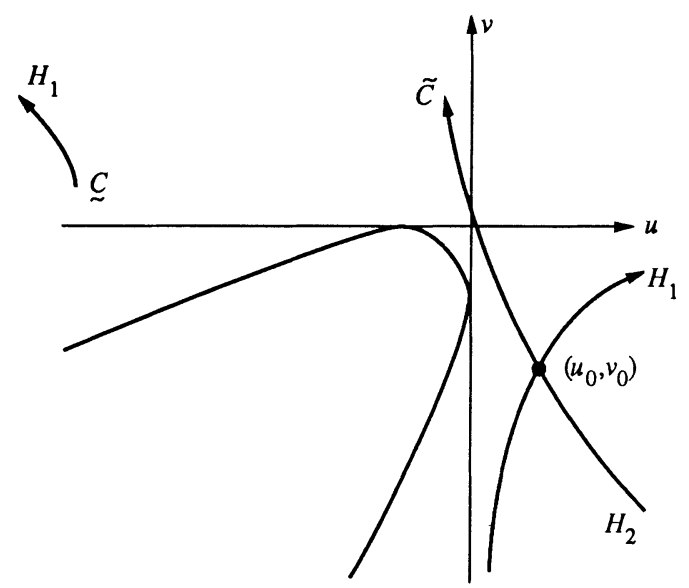

FIGURE 2.5

hand, the graph of the Hugoniot loci is shown as in Figure $2.4_{u_{0}}$ when $u_{0} \rightarrow 0$ which can be used to claim the continuity of the distribution as $\left(u_{0}, v_{0}\right)$ across the ray $u=0, v>-(a-1)$. Where the ray $u=0, v \geq-(a-1)$ belongs to $\mathrm{H}_{2}$

$$
v_{0}<-(a-1) \text {. }
$$

$H_{1}$ is specified by (2.8), it turns out that $v$ tends to $0^{-}$as $u \rightarrow+\infty$ while $v$ tends to $-\infty$ as $u \rightarrow 0$. Moreover, $H_{1}$ is not defined for $u^{(M)}\left(v_{0}\right)<$ $u<0$ but is defined again for $u<u^{(m)}\left(v_{0}\right)$, as shown in Figure 2.5. In fact, suppose $H_{1}$ be defined for $u^{(M)}\left(v_{0}\right)<u<0$, then $H_{1}$ should be given by (2.8) which shows that $v>0$ along $H_{1}$ when $0>u>u^{(M)}\left(v_{0}\right)$ since $-\left(u+v_{0}+a-1\right)-\sqrt{\left(v_{0}-u+a-1\right)^{2}+4(a-1) u}>0$ and $u-2 u_{0}-v_{0}+1-$ $a-\sqrt{\left(v_{0}-u+a-1\right)^{2}+4(a-1) u}<0$ for $0>u>u^{(M)}\left(v_{0}\right)$. Furthermore, $\sigma_{1} \rightarrow a$ and $v \rightarrow+\infty$ as $u \rightarrow 0^{-}$from (2.4.1) and (2.5.1). On the other hand, however, $\sigma_{1} \rightarrow-\infty$ as $v \rightarrow+\infty$ by (2.6.1), which gives a contradiction. 


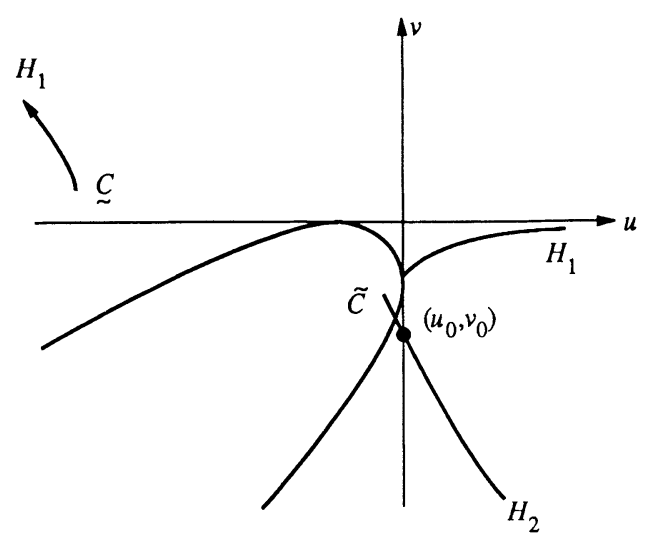

FIGURE $2.5_{u_{0}}$

For $\mathrm{H}_{2}$, it is more convenient to use (2.6.2) and (2.7.2) which provides

$$
v=v_{0} \frac{u-2 u_{0}-v_{0}+1-a+\sqrt{\left(v_{0}-u+a-1\right)^{2}+4(a-1) u}}{-\left(u+v_{0}+a-1\right)+\sqrt{\left(v_{0}-u+a-1\right)^{2}+4(a-1) u}} .
$$

It is easy to show by (2.14) that $H_{2}$ is defined for $u>u^{(M)}\left(v_{0}\right)$ and $v=v_{\widetilde{C}}$ at $u=u^{(M)}\left(v_{0}\right), H_{2}$ being tangent to the line $u=u^{(M)}\left(v_{0}\right)$ there. However, it can be shown similarly to $H_{1}$ for $u^{(M)}\left(v_{0}\right)<u<0$ that $H_{2}$ is not defined for $u<u^{(m)}\left(v_{0}\right)$. In fact, suppose $H_{2}$ be defined for $u<u^{(m)}\left(v_{0}\right)$, then (2.6.2) and (2.14) show that $\sigma_{2} \rightarrow 1$ and $v \rightarrow 0^{+}$as $u \rightarrow-\infty$. On the other hand, $\sigma_{2} \rightarrow u_{0}+a>a>1$ as $v \rightarrow 0$ by (2.4.2) which gives the contradiction. Since $\mathrm{H}_{2}$ is a monotonic function of $u$ for $u>u^{(M)}\left(v_{0}\right), H_{2}$ can be considered as a function of $v$ defined on $\left(-\infty, v_{\widetilde{C}}\right.$.

Remark 2.3. The graph of the Hugoniot loci varies continuously to the graph shown in Figure $2.5_{u_{0}}$ as $u_{0} \rightarrow 0$. Where the ray $u=0, v \leq-(a-1)$ belongs to $H_{1}$.

Case 3. $u_{0}<0, v_{0}>0$.

There are two cases: $u_{0}>-(a-1)$ or $u_{0}<-(a-1)$. Similar to Case 2, $H_{i}$ can be defined, as shown in Figures 2.6 and 2.7, respectively, where the two roots of $\left(v-u_{0}+a-1\right)^{2}+4(a-1) u_{0}=0$ are denoted by $v^{(M)}\left(u_{0}\right)$ and $v^{(m)}\left(u_{0}\right)$, respectively, namely

$$
\begin{gathered}
v^{(M)}\left(u_{0}\right)=-\left(\sqrt{a-1}-\sqrt{-u_{0}}\right)^{2}, \\
v^{(m)}\left(u_{0}\right)=-\left(\sqrt{a-1}+\sqrt{-u_{0}}\right)^{2} .
\end{gathered}
$$




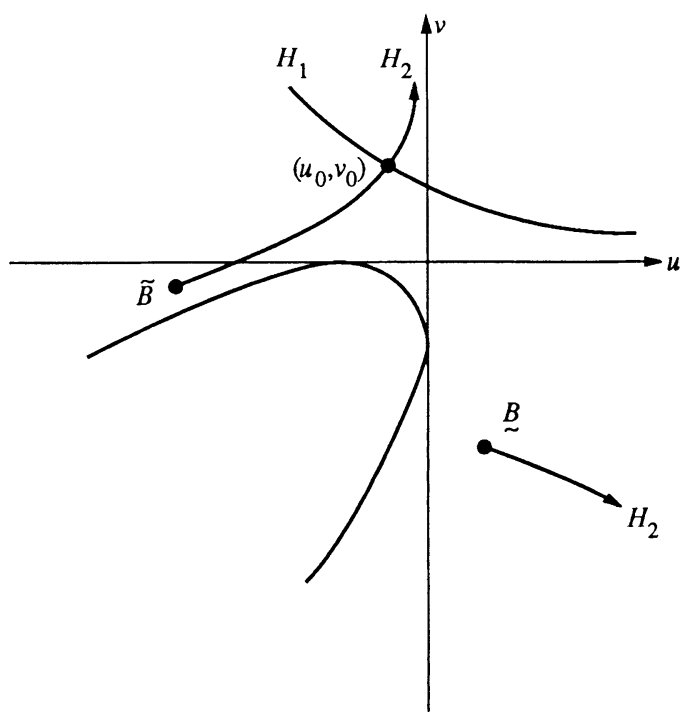

FigURE 2.6

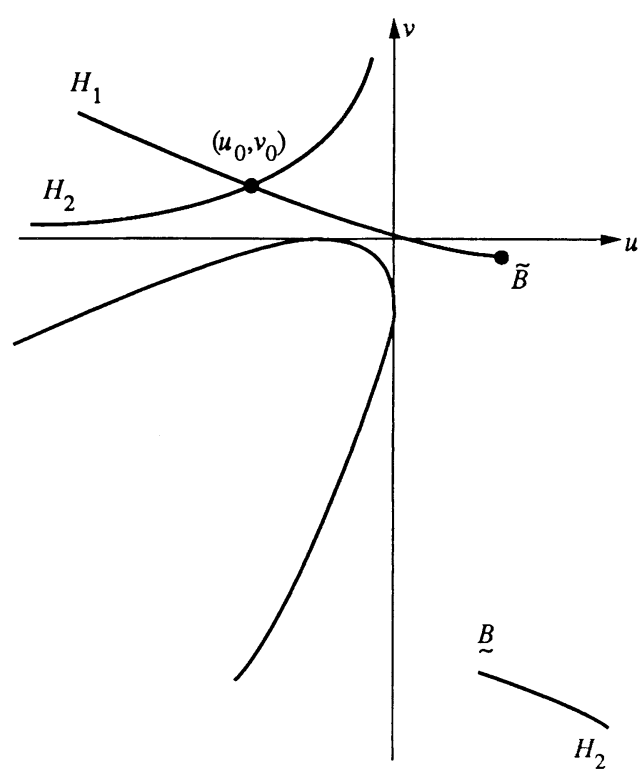

FIGURE 2.7

$H_{2}$ is defined for $v>v^{(M)}\left(u_{0}\right)$ and stops at $\widetilde{B} \equiv\left(u_{\widetilde{B}}, v^{(M)}\left(u_{0}\right)\right)$ when $u_{0}>$ $-(a-1)$ where

$$
u_{\widetilde{B}}=\frac{\sqrt{-u_{0}}}{\sqrt{-u_{0}}-\sqrt{a-1}} v_{0}-\sqrt{(a-1)\left(-u_{0}\right)}
$$

while $H_{1}$ is defined for $v>v^{(M)}\left(u_{0}\right)$ and stops at $\widetilde{B}$ when $u_{0}<-(a-1)$. Moreover, $H_{2}$ can be defined for $v<v_{\sim}^{B}=v^{(m)}\left(u_{0}\right)$ for each case, originating 


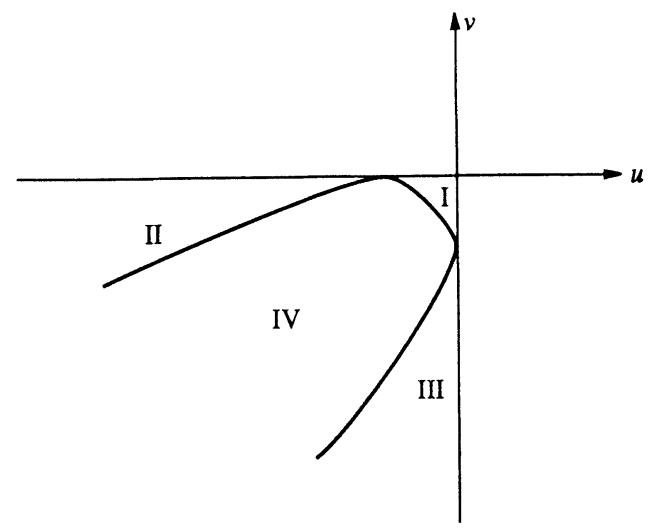

FIGURE 2.8

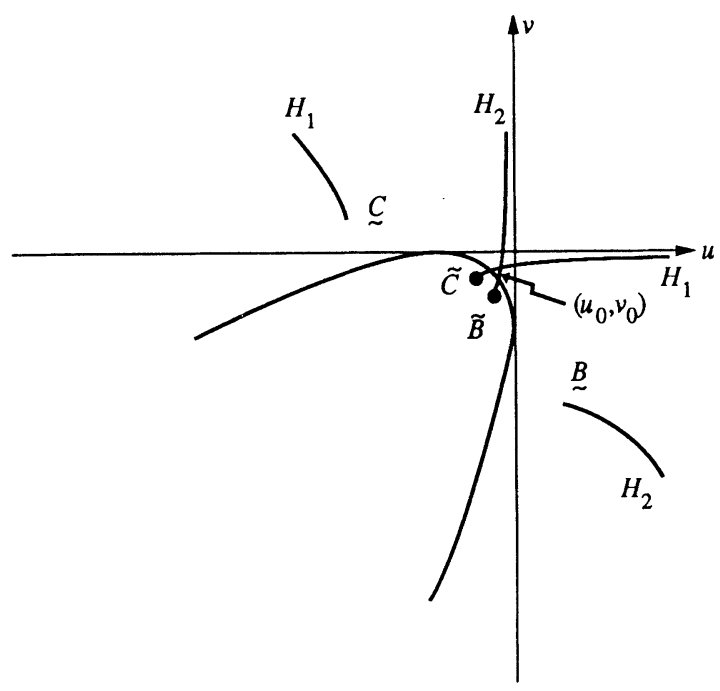

FigURE 2.9

at the point $\underset{\sim}{B} \equiv\left(u_{\sim}, v_{\sim}\right)$ where

$$
u_{\stackrel{B}{D}}=\frac{\sqrt{-u_{0}}}{\sqrt{u_{0}}+\sqrt{a-1}} v_{0}+\sqrt{(a-1)\left(-u_{0}\right)} .
$$

Similar to Case 2, the graph of Hugoniot loci changes continuously as $v_{0} \rightarrow 0$ for $u_{0}>-(a-1)$ or $u_{0}<-(a-1)$ respectively.

To consider the case $u_{0}<0, v_{0}<0$, it is convenient to consider the domain $\{u<0, v<0\}$ as divided into four subdomains by the curve $\Delta(u, v)=0$. We shall denote such subdomains by I, II, III, IV as displayed in Figure 2.8.

Case 4. $\left(u_{0}, v_{0}\right) \in \mathrm{I}$.

It can be shown that $H_{1}$ is defined for $u \geq u^{(M)}\left(v_{0}\right)$ and $u \leq u^{(m)}\left(v_{0}\right)$ with end-point $\widetilde{C}$ and $\underset{\sim}{C}$ respectively while $H_{2}$ is defined for $v \geq v^{(M)}\left(u_{0}\right)$ 


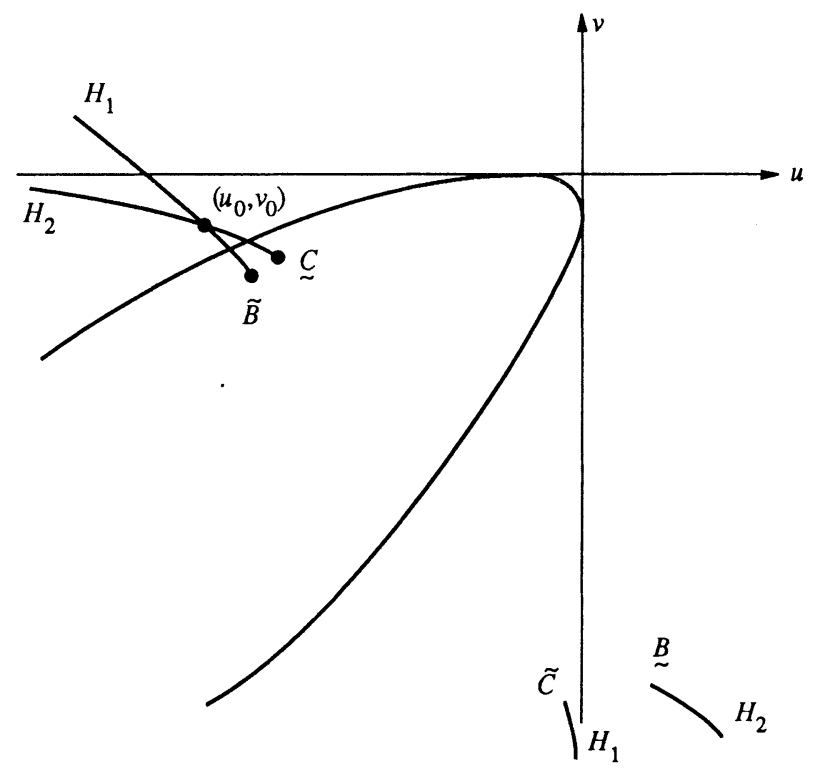

FIGURE 2.10

and $v \leq v^{(m)}\left(u_{0}\right)$ with end-point $\widetilde{B}$ and $\underset{\sim}{B}$ respectively, shown in Figure 2.9, where $\widetilde{C}$ and $\widetilde{B}$ approach the same point on the parabolic boundary $\Delta=0$ as $\left(u_{0}, v_{0}\right)$ approaches from the domain $\mathrm{I}$.

Case 5. $\left(u_{0}, v_{0}\right) \in \mathrm{II}$.

There are different subcases corresponding to $v_{0}<-(a-1)$ or $v_{0}>-(a-1)$.

(i) $v_{0}<-(a-1)$ : In this case, $H_{1}$ can be defined by (2.8) for $u \geq u^{(M)}\left(v_{0}\right)$ since $v_{\widetilde{C}}<v^{(m)}\left(u_{0}\right)$ but is only defined for $u \leq u_{\widetilde{B}}$ with $v=v^{(M)}\left(u_{0}\right)$ at $u=u_{\widetilde{B}}$ since $u^{(m)}\left(v_{0}\right)>u_{\widetilde{B}} . H_{2}$ can be defined by (2.9) for $v \leq v^{(m)}$ since $u_{\underset{\sim}{B}}>u^{(M)}\left(v_{0}\right)$ but is only defined for $0>v \geq v_{\underset{\sim}{C}}$ with $u=u^{(m)}\left(v_{0}\right)$ at $v=v_{C}$ since $v^{(M)}\left(u_{0}\right)<v_{\mathcal{C}}$. Moreover, it can be proved that $H_{2}$ is not defined for $v>0$ (see Figure 2.10) and $H_{1}$ is not defined for $u>0$. The points $\widetilde{B}$ and $\underset{\sim}{C}$ approach the same point on the parabolic boundary $\Delta=0$ as $\left(u_{0}, v_{0}\right)$ approaches from the domain II.

(ii) $v_{0}>-(a-1)$ : In this case $H_{1}$ can be defined by (2.8) for $u \leq u_{\widetilde{B}}$ with $v=v^{(M)}\left(u_{0}\right)$ at $u=u_{\widetilde{B}}$ since $u^{(m)}\left(v_{0}\right)>u_{\widetilde{B}}$. However, $H_{1}$ is not defined for $u>u^{(M)}\left(v_{0}\right)$. In fact, suppose $H_{1}$ defined for $u>u^{(M)}\left(v_{0}\right)$, then $H_{1}$ should be given by (2.8). It is easy to see that $v \rightarrow v_{\widetilde{C}}>0$ as $u \rightarrow u^{(M)}\left(v_{0}\right)$;

$$
v \rightarrow v_{0} \cdot \frac{u_{0}+v_{0}+a-1}{v_{0}+a-1}>0 \quad \text { as } u \rightarrow 0
$$

and $H_{1}$ should be defined for any $u>0$ with $v \rightarrow 0$ as $u \rightarrow+\infty$. This 


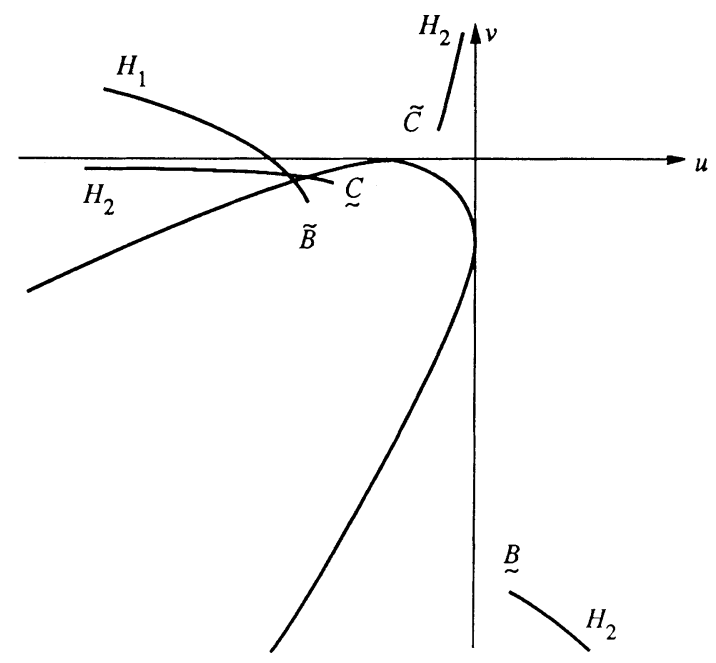

FIGURE 2.11

implies, by (2.6.1), that $\sigma_{1} \rightarrow u_{0}+a$ along $H_{1}($ as $u \rightarrow+\infty)$ and therefore

$$
\frac{u-u_{0}}{v-v_{0}} \rightarrow-\frac{u_{0}}{u_{0}+a-1}<0
$$

by (2.7.1) which is impossible. Similar to the case when $v_{0}<-(a-1), H_{2}$ can be defined by (2.9) for $v \leq v^{(m)}$ and $0>v \geq v_{\mathcal{C}}$. Moreover, $H_{2}$ can be defined for $v \geq v_{\widetilde{C}}$ also along which $u \rightarrow 0^{-}$as $v \rightarrow+\infty$. (See Figure 2.11 where the states $\widetilde{B}$ and $\underset{\sim}{C}$ approach the same state on the boundary $\Delta=0$ as $\left(u_{0}, v_{0}\right)$ approaches from the domain II.)

Remark 2.4. The graph of Hugoniot loci changes continuously to the graph shown in Figure $2.11_{v_{0}}$ as $v_{0} \rightarrow 0$ which is the same as in Case 3 when $u_{0}<$ $-(a-1), v_{0} \rightarrow 0^{+}$. Where the ray $v=0, u \leq-(a-1)$ belongs to $H_{2}$.

Remark 2.5. It is obvious that the graph of Hugoniot loci does not change continuously when $\left(u_{0}, v_{0}\right)$ cross the ray $v=-(a-1), u \leq 0$. It will be shown in Case 6 and Case 7 that both the rays $v=-(a-1), v \leq 0 ; u=-(a-1)$, $v \leq 0$ play an essential role. When $\left(u_{0}, v_{0}\right)$ cross these rays, the distribution of Hugoniot loci changes rapidly.

Case 6. $\left(u_{0}, v_{0}\right) \in$ III .6

Similar to Case 5, there are different subcases corresponding to $u_{0}<$ $-(a-1)$ or $u_{0}>-(a-1)$. When $u_{0}<-(a-1), H_{1}$ can be defined by (2.8) for $0>u \geq u_{\underset{B}{B}}$ with $v=v^{(m)}\left(u_{0}\right)$ at $u=u_{\underset{B}{B}}$ and for $u \leq u^{(m)}\left(v_{0}\right)$; $H_{2}$ can be defined by (2.9) for $v \leq v_{\widetilde{C}}$ with $u=u^{(\widetilde{M})}\left(v_{0}\right)$ at $v=v_{\widetilde{C}}$ and for $0>v \geq v^{(M)}\left(u_{0}\right)$ (see Figure 2.12 where $\underset{\sim}{B}$ and $\widetilde{C}$ approach the same point as $\left(u_{0}, v_{0}\right)$ approaches the boundary $\Delta=0$ from the domain III). 


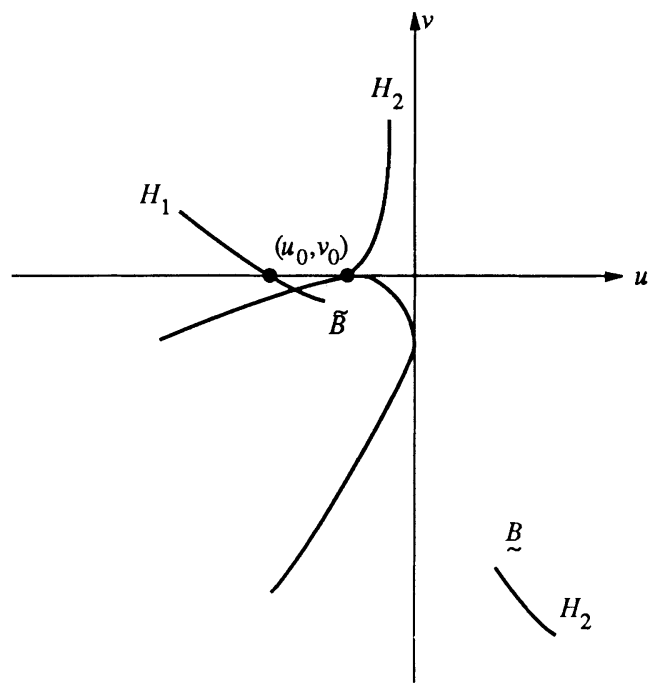

FIGURE $2.11_{v_{0}}$

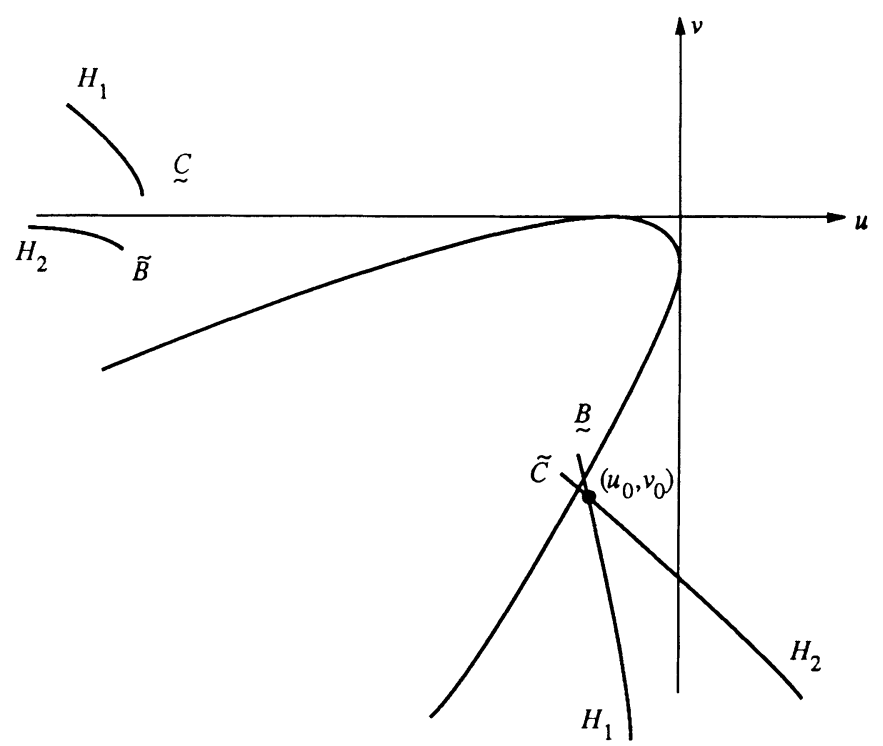

Figure 2.12 (where it is possible for $v_{\mathcal{C}}$ to be negative)

When $u_{0}>-(a-1): H_{1}$ can be defined by (2.8) for $u_{\underset{\sim}{B}} \leq u<0$ with $v=v^{(m)}\left(u_{0}\right)$ at $u=u_{\underset{\sim}{B}}$ and for $u \leq u^{(m)}\left(v_{0}\right)$ also. Moreover, $H_{1}$ can be defined for $u \geq u_{\widetilde{B}}$ along which $v \rightarrow 0^{-}$as $u \rightarrow+\infty . H_{2}$ can be defined by (2.9) for $v \leq v_{\widetilde{C}}$ with $u=u^{(M)}\left(v_{0}\right)$ at $v=v_{\widetilde{C}}$ but is not defined for anywhere else. (See Figure 2.13 where $\underset{\sim}{B}$ and $\widetilde{C}$ approach the same point as $\left(u_{0}, v_{0}\right)$ approaches the boundary $\Delta=0$ from the domain III.) 


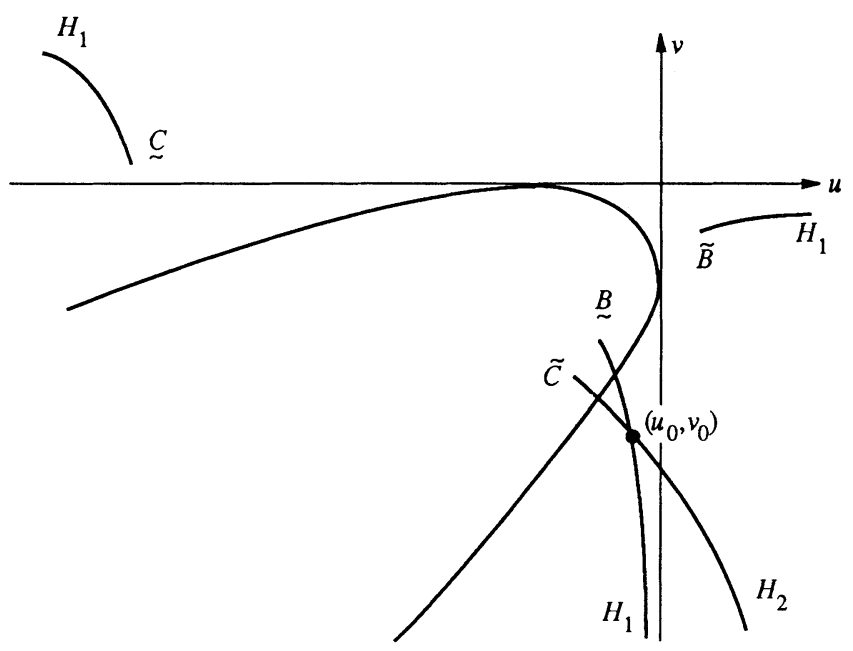

FigURE 2.13

Case 7. $\left(u_{0}, v_{0}\right) \in \mathrm{IV}$.

There are four subcases. The $H_{i}$ can be defined as in Figures 2.14-2.17 respectively. We omit the details.

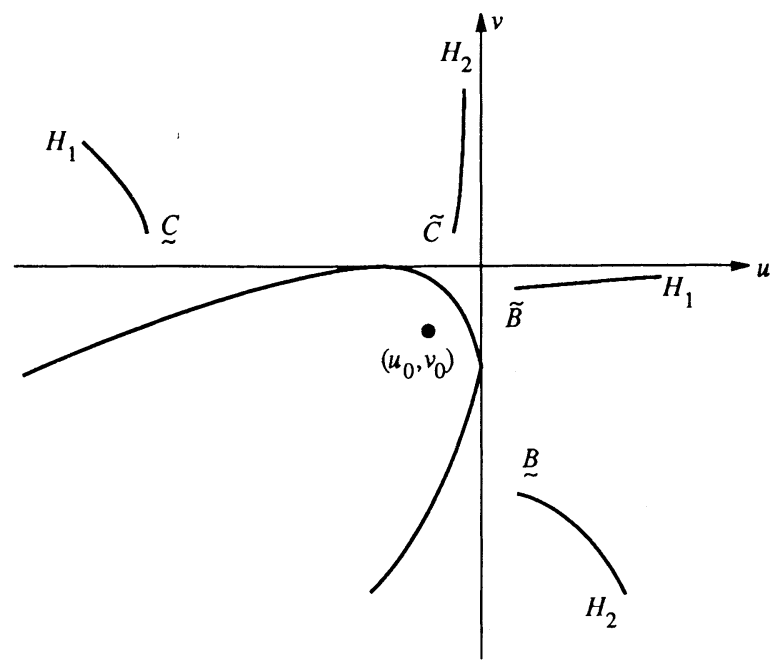

FIGURE 2.14. $v_{0}>-(a-1), v_{0}>-(a-1)$. Where $v_{\mathcal{C}}>0, u_{\underset{\sim}{B}}>0, u_{\widetilde{B}}$ and $u_{\widetilde{C}}$ may be positive or negative and the points $\widetilde{B}$ and $\widetilde{C}$ approach to $\left(u_{0}, v_{0}\right)$ as $\left(u_{0}, v_{0}\right)$ approaches the boundary $\Delta=0$. 


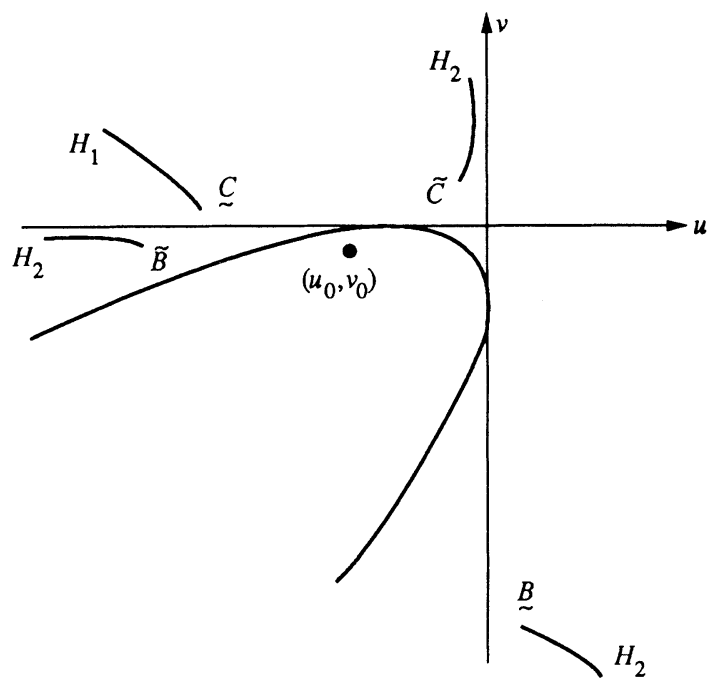

FIGURE 2.15. $v_{0}>-(a-1), u_{0}<-(a-1)$. Where $u_{\underset{\sim}{B}}>0, v_{\widetilde{C}}>0$, the sign of $v_{\underset{\sim}{C}}$ is not definite. $\underset{\sim}{C}$ and $\widetilde{B}$ approach $\left(u_{0}, v_{0}\right)$ as $\left(u_{0}, v_{0}\right)$ approaches the boundary $\Delta=0$.

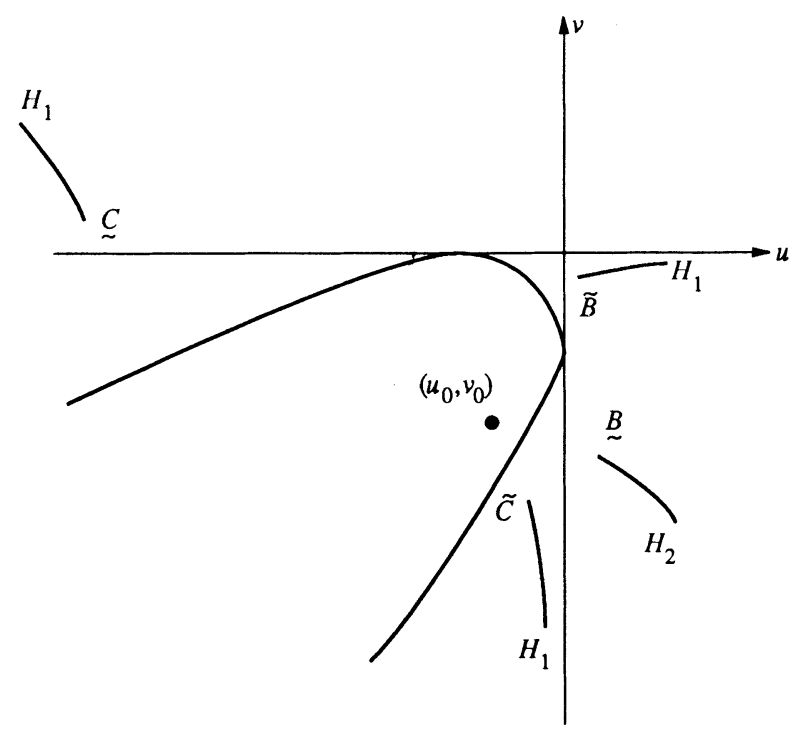

FIgURE 2.16. $v_{0}<-(a-1), u_{0}>-(a-1)$. Where $v_{C}>0, u_{\underset{\sim}{B}}>0$, the sign of $u_{\widetilde{B}}$ is not definite. $\underset{\sim}{B}$ and $\widetilde{C}$ approach $\left(u_{0}, v_{0}\right)$ as $\left(u_{0}, v_{0}\right)$ approaches the boundary $\Delta=0$.

It is clear from the above discussion that the distribution of the Hugoniot loci is stable as $\left(u_{0}, v_{0}\right)$ crosses the curve $\Delta=0$ except the points where the curve $\Delta=0$ intersects the curve $u=-(a-1)$ or $v=-(a-1)$. 


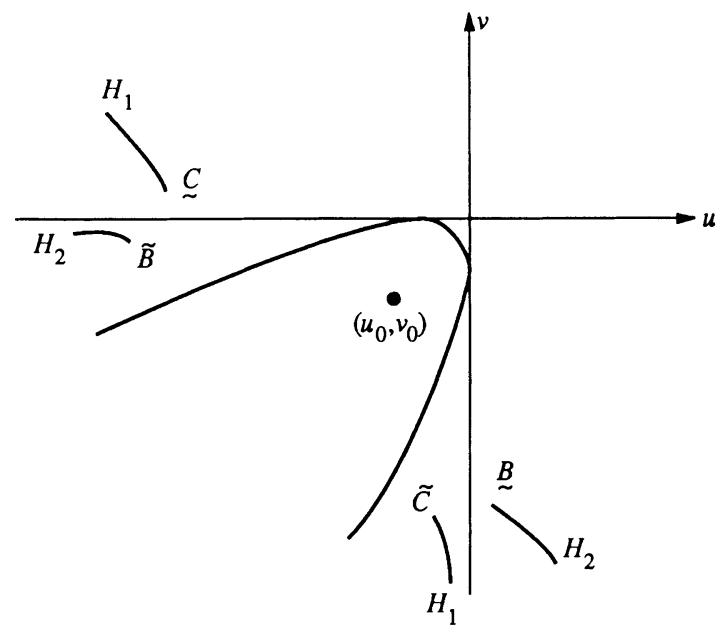

FIGURE 2.17. $v_{0}<-(a-1), u_{0}<-(a-1)$. Where the sign of both $v_{\mathcal{C}}$ and $u_{B}$ is not definite. $\widetilde{B}$ and $\underset{\sim}{C}$ approach $\left(u_{0}, v_{0}\right)$ as $\left(\tilde{u_{0}}, v_{0}\right)$ approaches boundary $\Delta=0$.

Remark 2.6. The distribution of Hugoniot loci varies continuously as $\left(u_{0}, v_{0}\right)$ varies on the whole plane $(u, v)$ except the rays $v=-(a-1), u \leq 0$, or $u=-(a-1), v \leq 0$.

For handling the elliptic domain in our system (1.1), we combine and generalize the classical Lax shock criterion and Liu-Oleinik shock (E) criterion for strictly hyperbolic system to introduce the generalized entropy condition as follows:

For any given $\left(u_{-}, v_{-}\right),\left(u_{+}, v_{+}\right) \in H_{1}\left(u_{-}, v_{-}\right)$is said to fulfil the generalized entropy condition (G.E.C.) if either

I. For any $u$ between $u_{-}$and $u_{+}$where $\sigma_{1}$ is defined, it holds that $\sigma_{1}\left(u ; u_{-}, v_{-}\right) \geq \sigma_{1}\left(u_{+} ; u_{-}, v_{-}\right)$or

II. $\sigma_{1}\left(u ; u_{-}, v_{-}\right)$is nonincreasing with respect to $\left|u-u_{-}\right|$for all $u \in \mathscr{I}_{+,-}$ for which it is defined, whereby

$$
\mathscr{I}_{+,-}= \begin{cases}{\left[u_{-}, u_{+}\right)} & \text {if } u_{-}<u_{+} \\ \left(u_{+}, u_{-}\right] & \text {if } u_{+}<u_{-}\end{cases}
$$

(Note that II implies I only in case $H_{1}$ is a connected curve.)

Similarly, $\left(u_{+}, v_{+}\right) \in H_{2}\left(u_{-}, v_{-}\right)$is said to fulfil the G.E.C. if either

I. For any $v$ between $v_{-}$and $v_{+}$where $\sigma_{2}$ is defined, it holds $\sigma_{2}\left(v ; u_{-}, v_{-}\right)$ $\geq \sigma_{2}\left(v_{+} ; u_{-}, u_{-}\right)$or

II. $\sigma_{2}\left(v ; u_{-}, v_{-}\right)$is nonincreasing with respect to $\left|v-v_{-}\right|$for all $v \in \mathscr{I}_{+,-}$ for which it is defined, whereby

$$
\mathscr{I}_{+,-}= \begin{cases}{\left[v_{-}, v_{+}\right)} & \text {if } v_{-}<v_{+} \\ \left(v_{+}, v_{-}\right] & \text {if } v_{+}<v_{-}\end{cases}
$$




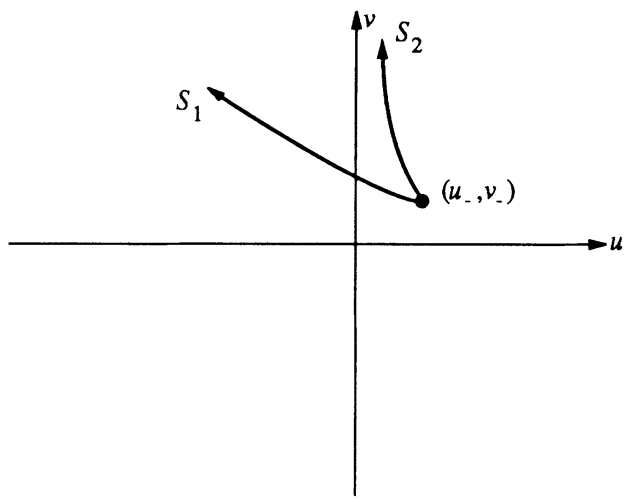

FIGURE 2.18

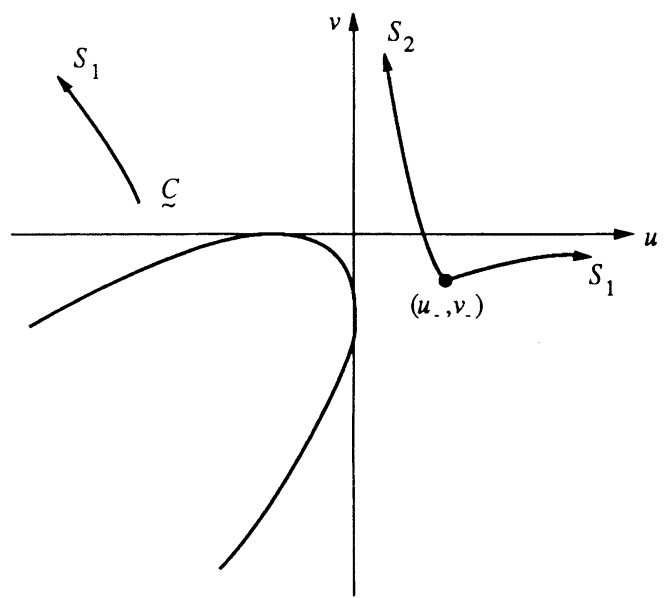

FIGURE 2.19

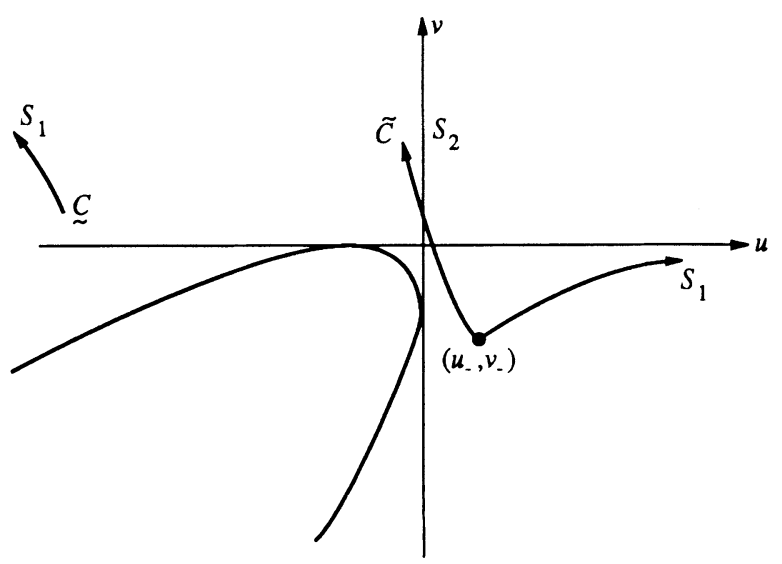

FigURE 2.20 
Remark 2.7. The G.E.C. can be used for the system in which the Hugoniot locus can be parametrized (not necessary by $u$ or $v$ with a slight change in the statement then). Denote the set of states, belonging to $H_{i}$ and satisfying the above G.E.C. by $S_{i}$. For showing $S_{i}$, we need a detailed distinction of cases again.

Case 1. $u_{-}>0, v_{-}>0$.

$S_{1}$ is defined by (2.8) for $u \leq u_{-}$on which $\sigma_{1}$ decreases as $u$ decreasing. $S_{2}$ is defined by (2.9) for $v \geq v_{-}$on which $\sigma_{2}$ decreases as $v$ increasing and $\sigma_{2} \rightarrow a$ as $v \rightarrow+\infty$ (see Figure 2.18) where the direction shows the direction along which $\sigma$ is decreasing.

Case 2. $u_{-}>0, v_{-}<0$.

When $0>v_{-}>-(a-1), S_{1}$ is defined by (2.8) for $u \geq u_{-}$, moreover, it is easy to show that $\sigma_{1}$ decreases on $S_{1}$ as $u$ increases and $\sigma_{1} \rightarrow 1$ as $u \rightarrow+\infty ; S_{1}$ is defined as well for $u \leq u^{(m)}\left(v_{-}\right)$, correspondingly $\sigma_{1}$ decreases as $u$ decreases (see Figure 2.19). $S_{2}$ is defined by (2.9) for $v \geq v_{-}$, here $\sigma_{2}$ decreases as $v$ increases and $\sigma_{2} \rightarrow a$ as $v \rightarrow+\infty$ (Figure 2.19).

When $v_{0}<-(a-1), S_{1}$ is defined by (2.8) for $u \geq u_{-}$and $u \leq u^{(m)}\left(v_{-}\right)$, the same as the case when $0>v_{0}>-(a-1) . S_{2}$ is defined by (2.9) for $v_{\widetilde{C}} \geq v \geq v_{-}$(see Figure 2.20) and $\sigma_{2}$ decreases on $S_{2}$ as $v$ increases.

Case 3. $u_{-}<0, v_{-}>0$.

$S_{i}$ is shown in Figures 2.21 and 2.22 corresponding to $u_{-}>-(a-1)$ or $u_{-}<-(a-1)$ respectively where the state $\underset{\sim}{B}$ belongs to $\bar{S}_{2}$ in both cases because of II in the G.E.C.

Case 4. $\left(u_{-}, v_{-}\right) \in \mathrm{I}$.

$S_{1}$ is defined by (2.8) for $u \geq u_{-}$and $u \leq u^{(m)}\left(v_{-}\right)$while $S_{2}$ is defined for $v^{(M)}\left(u_{-}\right) \leq v \leq v_{-}$. Moreover, $\underset{\sim}{B} \in S_{2}$ (see Figure 2.23).

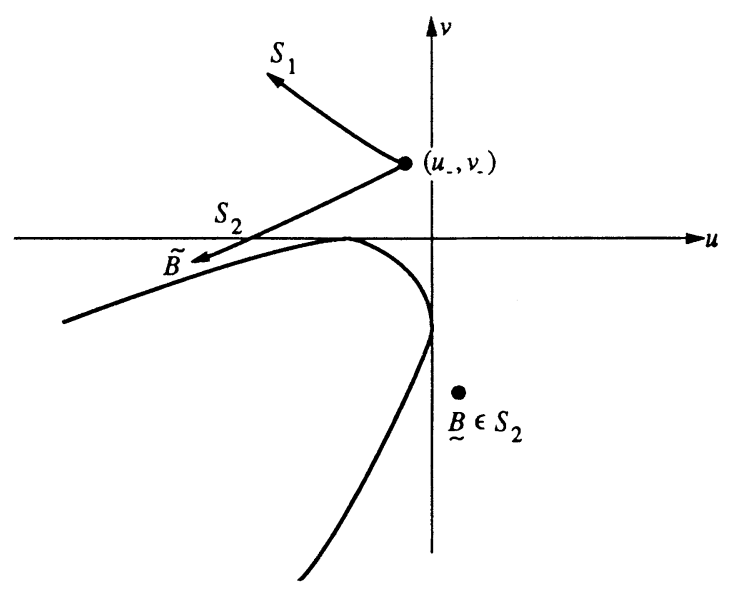

FIGURE 2.21 


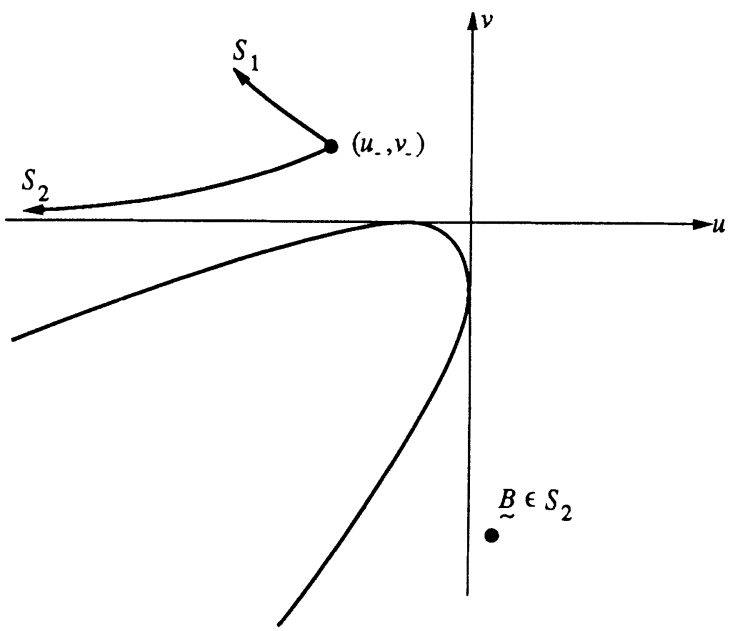

FIGURE 2.22

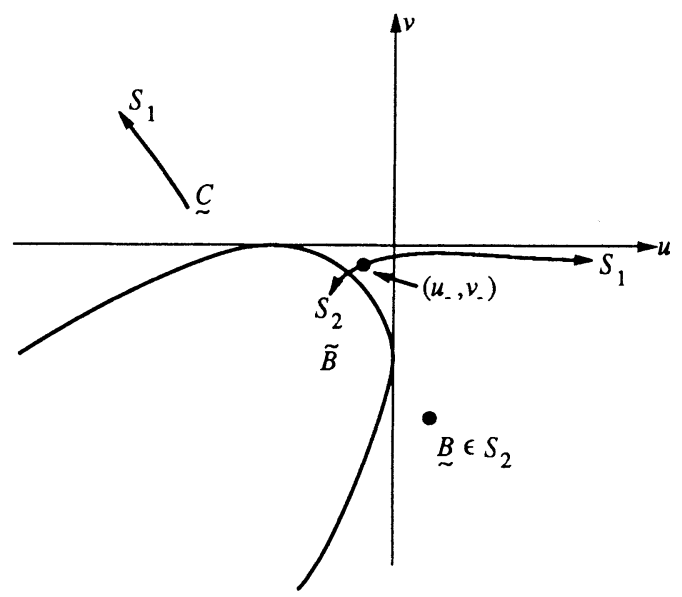

FIGURE 2.23

Case 5. $\left(u_{-}, v_{-}\right) \in \mathrm{II}$.

When $v_{-}<-(a-1), S_{1}$ is defined by (2.8) for $u \leq u_{-}, \sigma_{1}$ decreases on $S_{1}$ as $u$ decreases (see Figure 2.24). $S_{2}$ is defined by (2.9) for $v_{\mathcal{C}} \leq v \leq v_{-}$, $\sigma_{2}$ decreases on $S_{2}$ as $v$ decreases and the state $\underset{\sim}{B}$ belongs to $S_{2}$ also (Figure 2.24).

When $v_{-}>-(a-1), S_{1}$ is defined by (2.8) for $u \leq u_{-} . S_{2}$ is defined by (2.9) for $v_{\mathcal{C}} \leq v \leq v_{-}$and the state $\underset{\sim}{B}$ belongs to $S_{2}$ also (Figure 2.25).

Case 6. $\left(u_{-}, v_{-}\right) \in \mathrm{III}$.

When $u_{-}<-(a-1), S_{1}$ is defined by $(2.8)$ for $u \leq u^{(m)}\left(v_{-}\right)$and $u_{-} \leq$ $u<0$ (see Figure 2.26). $S_{2}$ is defined by (2.9) for $v_{-} \leq v \leq v_{\widetilde{C}}, \widetilde{B} \in S_{2}$ also. 


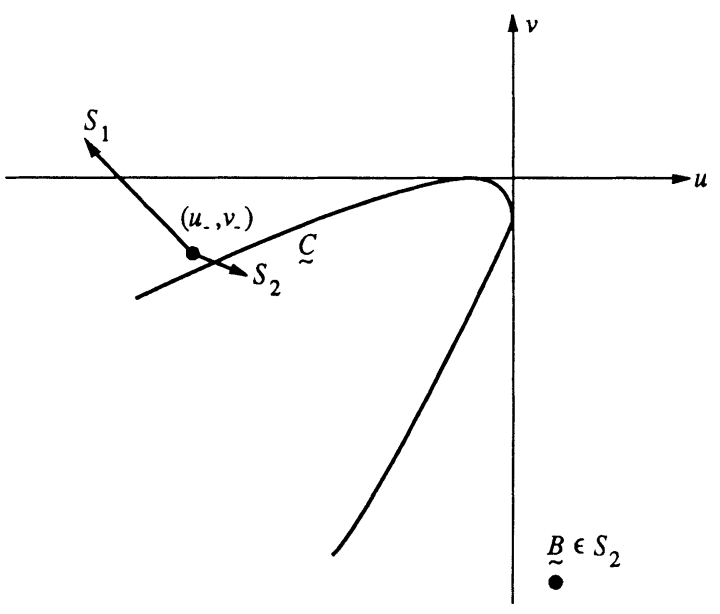

FIGURE 2.24

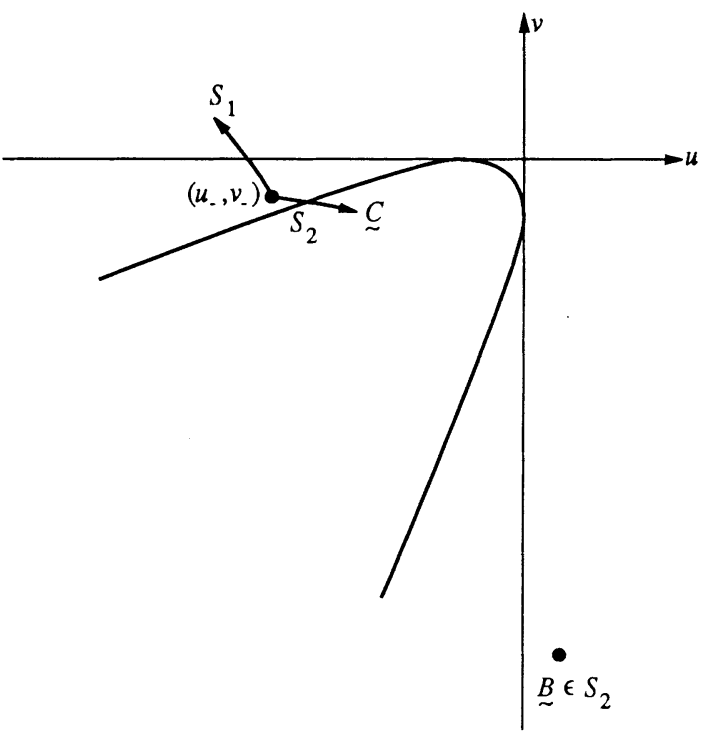

FiguRE 2.25

When $u_{-}>-(a-1), S_{1}$ is defined by $(2.8)$ for $u \leq u^{(m)}\left(v_{-}\right), u_{-} \leq u \leq 0$ and $u \geq u_{\widetilde{B}} . S_{2}$ is defined by (2.9) for $v_{-} \leq v \leq v_{\widetilde{C}}$ (see Figure 2.27).

Case 7. $\left(u_{-}, v_{-}\right) \in \mathrm{IV}$.

Corresponding to different cases in Figures 2.14-2.17, the $S_{i}^{\prime}$ are shown in Figures 2.28-2.31 respectively.

Remark 2.8. The distribution of the set $S_{i}\left(u_{-}, v_{-}\right)$varies continuously when $\left(u_{-}, v_{-}\right)$varies in the plane $(u, v)$ except $\left(u_{-}, v_{-}\right)$across the curves $u=0$; $v=0$ and the rays $u=-(a-1), v \leq 0 ; v=-(a-1), u \leq 0$. 


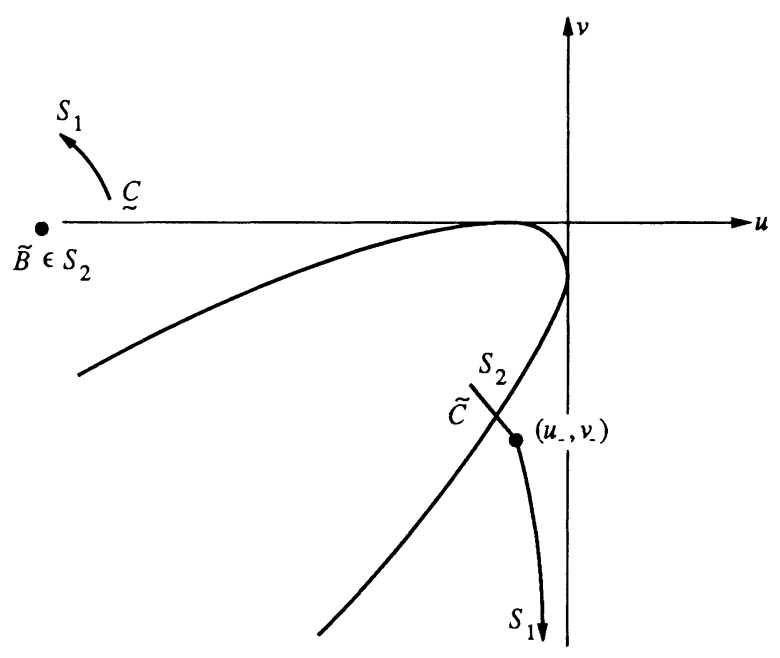

FIGURE 2.26

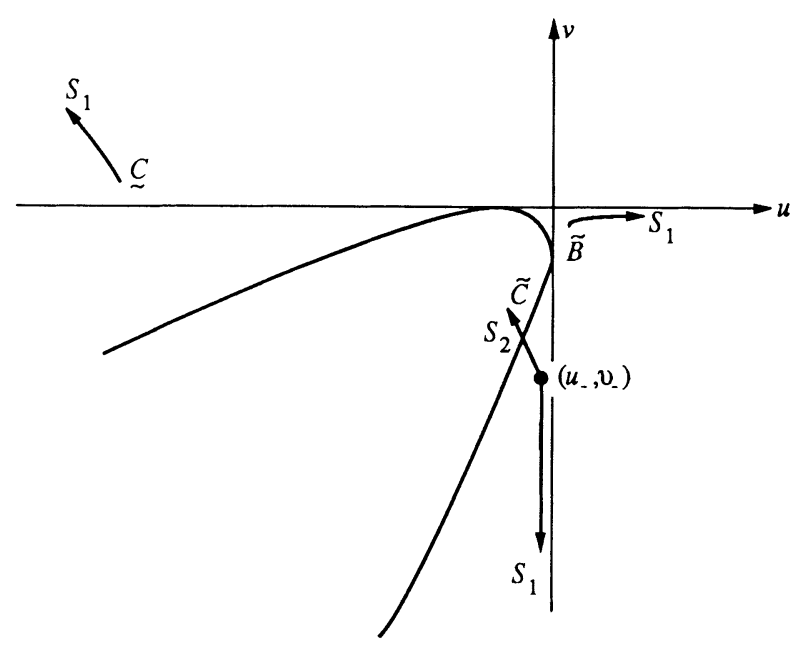

FIGURE 2.27

A complete classification concerning the relation between the speed of the admissible shock and the characteristic associated is proved in $\left[\mathrm{Hs}_{2}\right]$ which says that when both $U_{+}$and $U_{-}$are in the hyperbolic region then

1. Any state $U_{+}^{+}$located on $S_{i}\left(U_{-}\right)$, connected to the state $U_{-}$, supplies an admissible shock satisfying the Lax condition, namely

$$
\lambda_{1}\left(U_{+}\right) \leq \sigma_{1}\left(U_{+} ; U_{-}\right) \leq \lambda_{1}\left(U_{-}\right), \quad \sigma_{1}\left(U_{+} ; U_{-}\right) \leq \lambda_{2}\left(U_{+}\right)
$$

for $U_{+}=\left(u_{+}, v_{+}\right) \in S_{1}\left(U_{-}\right)$or

$$
\lambda_{2}\left(U_{+}\right) \leq \sigma_{2}\left(U_{+} ; U_{-}\right) \leq \lambda_{2}\left(U_{-}\right), \quad \lambda_{1}\left(U_{-}\right) \leq \sigma_{2}\left(U_{+} ; U_{-}\right)
$$

for $U_{+}=\left(u_{+}, u_{+}\right) \in S_{2}\left(U_{-}\right)$. 


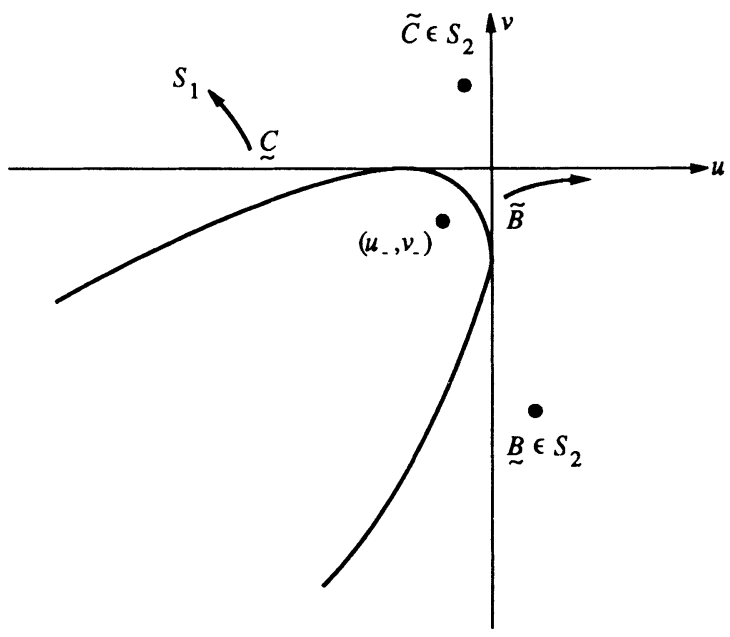

FiguRE 2.28. $v_{-}>-(a-1), u_{-}>-(a-1)$. Two branches of $S_{1}$, two isolated points $\widetilde{C}$ and $\underset{\sim}{B}$ belonging to $S_{2}$

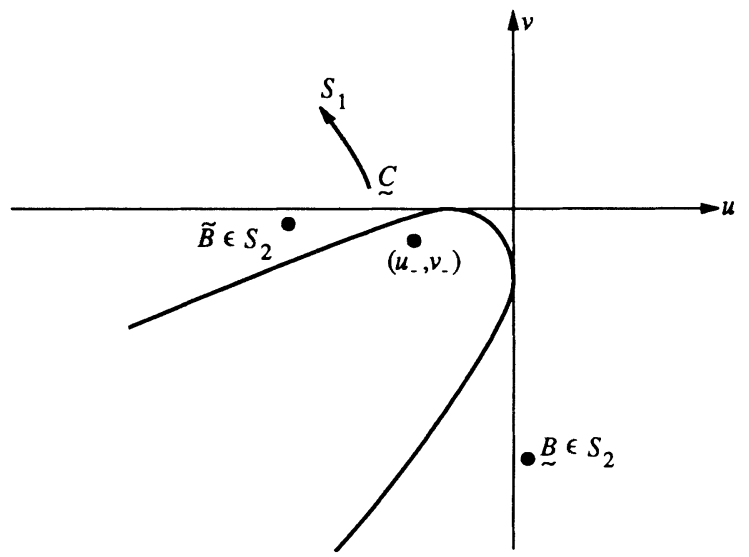

FIGURE 2.29. $v_{-}>-(a-1), u_{-}<-(a-1)$. One branch of $S_{1}$, two isolated points $\widetilde{B}$ and $\underset{\sim}{B}$ belonging to $S_{2}$.

2. Any state $U_{+}$located on $S_{1}\left(U_{-}\right)$with ending point $\underset{\sim}{C}$ or $\widetilde{B}$, disconnected to $U_{-}$, supplies an admissible shock satisfying the Lax condition (2.19).

3. When the state $U_{+}$takes the isolated state $\widetilde{B} \in S_{2}\left(U_{-}\right)$, the admissible shock agrees with the Lax condition on the number of characteristics whichenter or leave the shock but violates the Lax condition on the style, namely

$$
\sigma\left(\widetilde{B} ; U_{-}\right)<\lambda_{1}\left(U_{-}\right), \quad \lambda_{1}(\widetilde{B})<\sigma_{2}\left(\widetilde{B} ; U_{-}\right)<\lambda_{2}(\widetilde{B}) .
$$




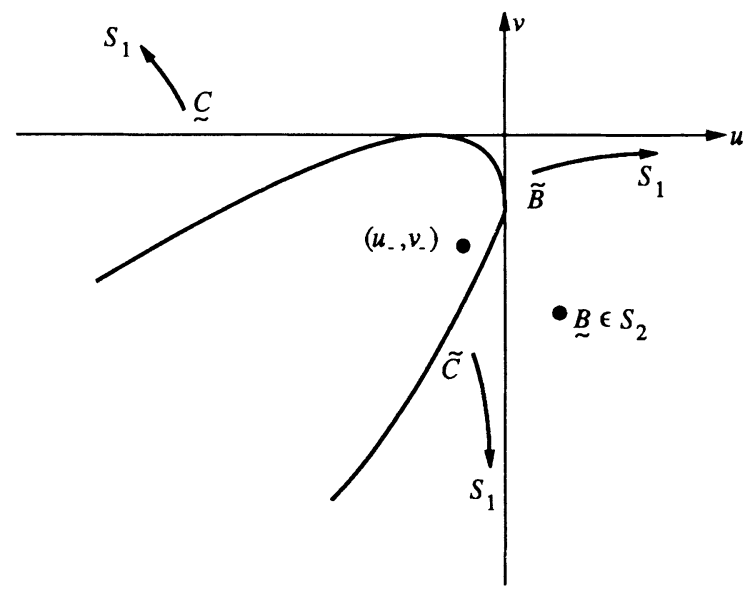

FigURE 2.30. $v_{-}<-(a-1), u_{-}>-(a-1)$. Three branches of $S_{1}$, one isolated point $\underset{\sim}{B} \in S_{2}$

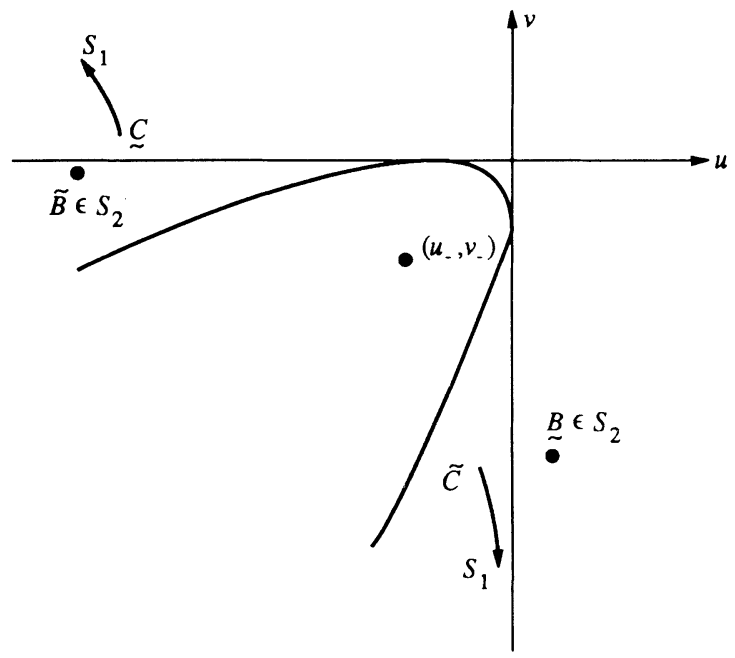

FIGURE 2.31. $v_{-}<-(a-1), u_{-}<-(a-1)$. Two branches of $S_{1}$, two isolated points $\widetilde{B} \in S_{2}$ and $\underset{\sim}{B} \in$ $S_{2}$.

4. When the state $U_{+}$takes the isolated state $\underset{\sim}{B} \in S_{2}\left(U_{-}\right)$, the admissible shock violates the Lax condition on both the numbers and the style. Namely

$$
\sigma_{2}\left(\underset{\sim}{B} ; U_{-}\right)>\lambda_{2}\left(U_{-}\right), \quad \lambda_{1}(\underset{\sim}{B})<\sigma_{2}\left(\underset{\sim}{B} ; U_{-}\right)<\lambda_{2}(\underset{\sim}{B}) .
$$

\section{EXISTENCE AND UNIQUENESS OF AN ADMISSIBLE WEAK SOLUTION}

Consider $(1.1)$ with initial data $(1.3)$ where $\left(u_{-}, v_{-}\right),\left(u_{+}, v_{+}\right)$are arbitrary states in the $(u, v)$ plane. Since both the system (1.1) and the initial data (1.3) 
are invariant under the transformation $x \rightarrow \alpha x, t \rightarrow \alpha t, \alpha>0$, we look for self-similar solution $u=u(\xi), v=v(\xi)$, where $\xi=\frac{x}{t}$.

Definition 3.1. A single-valued function $(u(\xi), v(\xi))$ is called an admissible weak solution of (1.1) (1.3) if

I. It satisfies the boundary conditions $(u, v) \rightarrow\left(u_{\mp}, v_{\mp}\right)$ as $\xi \rightarrow \mp \infty$.

II. It is either a rarefaction wave or a constant state wherever it is smooth.

III. Any discontinuity satisfies the Rankine-Hugoniot condition (2.3) and the generalized entropy condition (G.E.C.) introduced in $\S 2$.

IV. The sum of the strength of all of the jumps takes the minimum values among all possible single-valued function $(u(\xi), v(\xi))$ satisfying (I)-(III). Here, the strength of a jump in a $S_{1}$ (respectively, $S_{2}$ ) wave is measured by the jump in $u$ (respectively, $v$ ).

Remark 3.1. The uniqueness in our problem (1.1) (3.1) fails without the requirement IV in the above definition.

Remark 3.2. For a pure hyperbolic system of conservation laws our generalized entropy condition becomes the well-known entropy (E) condition (Liu-Oleinik condition) and the last item in Definition 3.1 is not needed.

For any given $\left(u_{-}, v_{-}\right)$, we consider the set of all states which can be joined to $\left(u_{-}, v_{-}\right)$, on the right-hand side, by a single-valued function $(u(\xi), v(\xi))$ satisfying the items I-III in Definition 3.1 and consisting of the first kind of waves. Namely, it contains either a 1-discontinuity (the first family) satisfying III in Definition 3.1 or a 1-rarefaction wave (the first family) or a fan of such first kind waves. We denote this set by $\bar{W}_{1}\left(u_{-}, v_{-}\right)$which is a curve on a $(u, v)$ plane for given $\left(u_{-}, v_{-}\right)$but not necessarily connected. For each point $\left(u_{1}, v_{1}\right) \in \bar{W}_{1}\left(u_{-}, v_{-}\right)$, we determine the set of all states which can be joined to $\left(u_{1}, v_{1}\right)$, on the right-hand side, by a single-valued function $(u(\xi), v(\xi))$ satisfying the items I-III in Definition 3.1 and consisting of second kind waves. Namely, it contains either a 2-discontinuity satisfying III in Definition 3.1 or a 2-rarefaction wave or a fan of such second kind waves. We denote this set as $\bar{W}_{2}\left(u_{1}, v_{1}\right)$. This is not necessarily a curve anymore, as in the case of a purely hyperbolic system, indeed, $\bar{W}_{2}\left(u_{1}, v_{1}\right)$ may contain a family of curves. We then define the family of admissible wave curves $A \bar{W}_{2}$ from $\left\{\bar{W}_{2}\left(u_{1}, v_{1}\right)\right.$; $\left.\left(u_{1}, v_{1}\right) \in \bar{W}_{1}\left(u_{-}, v_{-}\right)\right\}$by using the item IV in Definition 3.1 and denote the resulting whole family by $\{A \bar{W}\}\left(u_{-}, v_{-}\right)$. Namely, $\{A \bar{W}\}\left(u_{-}, v_{-}\right)=$ $\left\{A \bar{W}_{2}\left(u_{1}, v_{1}\right) ;\left(u_{1}, v_{1}\right) \in \bar{W}_{1}\left(u_{-}, v_{-}\right)\right\}$.

In order to prove the existence and uniqueness of the admissible weak solution for the Riemann problem (1.1) (1.3), it suffices to show that for any given $\left(u_{-}, v_{-}\right)$, the family of $\{A \bar{W}\}\left(u_{-}, v_{-}\right)$can be defined which covers the whole plane $(u, v)$ univalently. We give a constructive proof in the following, which shows, at the same time, the structure of the solutions. We only give the details for two typical cases, the other cases can be discussed in a similar way.

Case 1. $v_{-}>0,0>u_{-}>-(a-1)$.

Using the characterization of rarefaction wave curves (shown in Figure 2.2), 
that of discontinuities satisfying the Rankine-Hugoniot condition and the G.E.C. and Definition 3.1 , the family $\{A \bar{W}\}\left(u_{-}, v_{-}\right)$can be defined as follows: $\bar{W}_{1}\left(u_{-}, v_{-}\right)$consists of $S_{1}\left(u_{-}, v_{-}\right)$for $u<u_{-}$and $R_{1}\left(u_{-}, v_{-}\right)$for $u \geq u_{-}$. For any $\left(u_{1}, v_{1}\right) \in \bar{W}_{1}\left(u_{-}, v_{-}\right)$with $u_{1}>0$, the admissible second wave curve $A \bar{W}_{2}\left(u_{1}, v_{1}\right)$ is defined as $R_{2}\left(u_{1}, v_{1}\right)$ for $v \leq v_{1}$ while $S_{2}\left(u_{1}, v_{1}\right)$ for $v>v_{1}$. For any $\left(u_{1}, v_{1}\right) \in \bar{W}_{1}\left(u_{-}, v_{-}\right)$with $-(a-1)<u_{1}<0$, the state $\widetilde{B}\left(u_{1}, v_{1}\right)$ is defined (see the formula (2.15), (2.17), replacing $\left(u_{1}, v_{1}\right)$ for $\left(u_{0}, v_{0}\right)$ there), and give rise to the curve $C(\widetilde{B})$ when the state $\left(u_{1}, v_{1}\right)$ varies along $\bar{W}_{1}\left(u_{-}, v_{-}\right)$from $u_{1}=0$ to $u_{1}=-(a-1)$. It turns out that the curve $C(\widetilde{B})$, starting from the state $\{u=0, v=-(a-1)\}$, is monotone with nonpositive slope and $u \rightarrow-\infty, v \rightarrow 0$ as $u_{1} \rightarrow-(a-1)$. Let us denote the state $\{u=0, v=-(a-1)\}$ by $Q$, the state $\{v=0, u=-(a-1)\}$ by $P$. As $\left(u_{2}, v_{2}\right)$ varies on the curve $C(\widetilde{B})$, the state $\underset{\sim}{B}\left(u_{2}, v_{2}\right)$ (see formula (2.16), (2.18), replacing $\left(u_{2}, v_{2}\right)$ for $\left(u_{0}, v_{0}\right)$ there) forms the curve $\widehat{C}(\underset{\sim}{B})$, starting from the state $Q$ and is tangent with the $v$-axis there. For any $\left(u_{1}, v_{1}\right) \in \bar{W}_{1}\left(u_{-}, v_{-}\right)$ with $-(a-1)<u_{1}<0$, we denote the state $\underset{\sim}{B}$ corresponding to $\widetilde{B}\left(u_{1}, v_{1}\right)$ by ${\underset{\sim}{B}}^{*}=\left(u_{1}^{*}, v_{1}^{*}\right)$. Namely replace $\widetilde{B}\left(u_{1}, v_{1}\right)$ for $\left(u_{0}, v_{0}\right)$ in the formula (2.16) (2.18) in order to obtain $u_{1}^{*}$ and $v_{1}^{*}$. Then the admissible second wave curve $A \bar{W}_{2}\left(u_{1}, v_{1}\right)$ is defined as $R_{2}\left(u_{1}, v_{1}\right)$ for $v \geq v_{1} ; S_{2}\left(u_{1}, v_{1}\right)$ for $v_{1}>v \geq v_{\widetilde{B}\left(u_{1}, v_{1}\right)} ; S_{2}\left(u_{1}^{*}, v_{1}^{*}\right)$ for $v_{\widetilde{B}\left(u_{1}, v_{1}\right)}>v \geq v_{1}^{*}$ and $R_{2}\left(u_{1}^{*}, v_{1}^{*}\right)$ for $v<v_{1}^{*}$ (see Figure 3.1). For any $\left(u_{1}, v_{1}\right) \in \bar{W}_{1}\left(u_{-}, v_{-}\right)$with $u_{1}<-(a-1)$, $A \bar{W}_{2}\left(u_{1}, v_{1}\right)$ is defined as $R_{2}\left(u_{1}, v_{1}\right)$ for $v \geq v_{1} ; S_{2}\left(u_{1}, v_{1}\right)$ for $0<v<v_{1}$ (see Figure 3.1).

Remark 3.3. As $\left(u_{1}, v_{1}\right)$ varies on $\bar{W}_{1}\left(u_{-}, v_{-}\right)$with $u_{1}<0$, the corresponding state $\underset{\sim}{B}\left(u_{1}, v_{1}\right)$ (see the formulae (2.16) (2.18), replacing $\left(u_{1}, v_{1}\right)$ for $\left(u_{0}, v_{0}\right)$ there $)$ can be shown to form the curve $C(\underset{\sim}{B})$, starting from the state $Q$ and located on the right-hand side of the curve $\widehat{C}(\underset{\sim}{B})$ (see Proposition 3.1). Thus, there is a curve $K\left(u_{1}, v_{1}\right)$ in the set of $\bar{W}_{2}\left(u_{1}, v_{1}\right)$ for $\left(u_{1}, v_{1}\right) \in$ $\bar{W}_{1}\left(u_{-}, v_{-}\right)$with $u_{1}<0$ such that $K\left(u_{1}, v_{1}\right)$ is defined as $S_{2}\left(\underset{\sim}{B}\left(u_{1}, v_{1}\right)\right)$ for $v_{1}>v \geq v_{\underset{\sim}{B}\left(u_{1}, v_{1}\right)} ; R_{2}\left(\underset{\sim}{B}\left(u_{1}, v_{1}\right)\right)$ for $v<v_{\underset{\sim}{B}\left(u_{1}, v_{1}\right)}$. However, the above curves $K\left(u_{1}, v_{1}\right)$ (for $v>v_{1}$ ) do not satisfy item IV in Definition 3.1 and therefore do not belong to the family $\{A \bar{W}\}\left(u_{-}, v_{-}\right)$(see Proposition 3.2).

Proposition 3.1. For any $\left(u_{1}, v_{1}\right) \in \bar{W}_{1}\left(u_{-}, v_{-}\right)$with $u_{1}<0$, the state $\underset{\sim}{B}\left(u_{1}, v_{1}\right)$ forms the curve $C(\underset{\sim}{B})$ which starts from the state $Q$ and is located on the right-hand side of the curve $\widehat{C}(\underset{\sim}{\mathbb{B}})$.

Proof. The state $\underset{\sim}{B}\left(u_{1}, v_{1}\right)$ is expressed by

$$
\left\{\begin{array}{l}
v_{B}=-\left(\sqrt{a-1}+\sqrt{-u_{1}}\right)^{2}, \\
{\underset{\sim}{u_{B}}}^{2}=\frac{\sqrt{-u_{1}}}{\sqrt{-u_{1}+\sqrt{a-1}}} v_{1}+\sqrt{(a-1)\left(-u_{1}\right)}
\end{array}\right.
$$

where $\left(u_{1}, v_{1}\right) \in \bar{W}_{1}\left(u_{-}, v_{-}\right)$with $u_{1}<0$. 


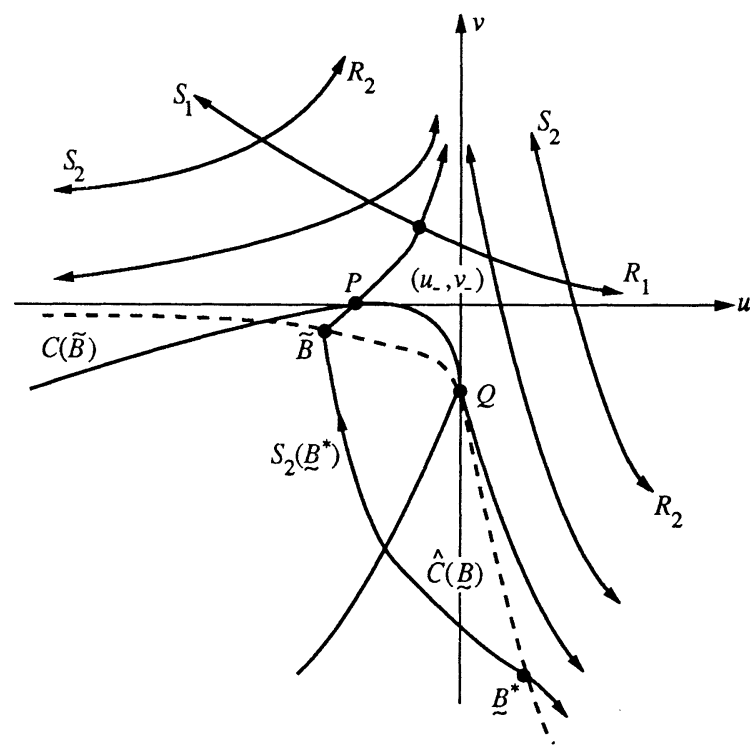

FIGURE 3.1

It is easy to see from (3.1) that $\underset{\sim}{v_{B}} \rightarrow-(a-1)$ and $\underset{\sim}{u_{B}} \rightarrow 0$ as $u_{1} \rightarrow 0^{-}$. While $v_{\underset{\mathcal{B}}{ }} \rightarrow-\infty$ and $u_{\underset{\sim}{B}} \rightarrow \infty$ as $\tilde{u}_{1} \rightarrow-\infty$.

Furthermore, it can be calculated that the slope along the curve $C(\underset{\sim}{B})$ takes the form

$$
\frac{d v_{\stackrel{B}{\sim}}}{d u_{\stackrel{B}{\sim}}}=\frac{\sqrt{a-1}+\sqrt{-u_{1}}}{\frac{-1}{2}\left[\frac{\sqrt{a-1}}{\left(\sqrt{\left.-u_{1}+\sqrt{a-1}\right)^{2}}\right.} \cdot v_{1}+\sqrt{a-1}\right]+\frac{{\sqrt{-u_{1}}}^{2}}{\sqrt{-u_{1}+\sqrt{a-1}} \cdot \frac{d v_{1}}{d u_{1}}}}
$$

where $\left(u_{1}, v_{1}\right) \in \bar{W}_{1}\left(u_{-}, v_{-}\right)$with $u_{1}<0$ and $d v_{1} / d u_{1}$ is the slope of the curve $\bar{W}_{1}\left(u_{-}, v_{-}\right)$at $\left(u_{1}, v_{1}\right)$. Therefore, the slope of the curve $C(\underset{\sim}{B})$ tends to

$$
\frac{\sqrt{a-1}}{\frac{-1}{2}\left[\frac{v_{1}}{\sqrt{a-1}}+\sqrt{a-1}\right]}
$$

as $u_{1} \rightarrow 0$ which is a finite negative number.

On the other hand, the states $\left(u_{2}, v_{2}\right)$ which forms the curve $\widehat{C}(\underset{\sim}{B})$ can be expressed as

$$
\left\{\begin{array}{l}
v_{B}=-\left(\sqrt{a-1}+\sqrt{-u_{2}}\right)^{2}, \\
u_{\sim}=\frac{\sqrt{-u_{2}}}{\sqrt{-u_{2}+\sqrt{a-1}}} v_{2}+\sqrt{(a-1)\left(-u_{2}\right)}
\end{array}\right.
$$

where $\left(u_{2}, v_{2}\right) \in C(\widetilde{B})$, namely

$$
\left\{\begin{array}{l}
v_{2}=-\left(\sqrt{a-1}-\sqrt{-u_{1}}\right)^{2}, \\
u_{2}=\frac{\sqrt{-u_{1}}}{\sqrt{-u_{1}-\sqrt{a-1}}} \cdot v_{1}-\sqrt{(a-1)\left(-u_{1}\right)}
\end{array}\right.
$$

with $\left(u_{1}, v_{1}\right) \in \bar{W}_{1}\left(u_{-}, v_{-}\right)$and $u_{1}<0$. 


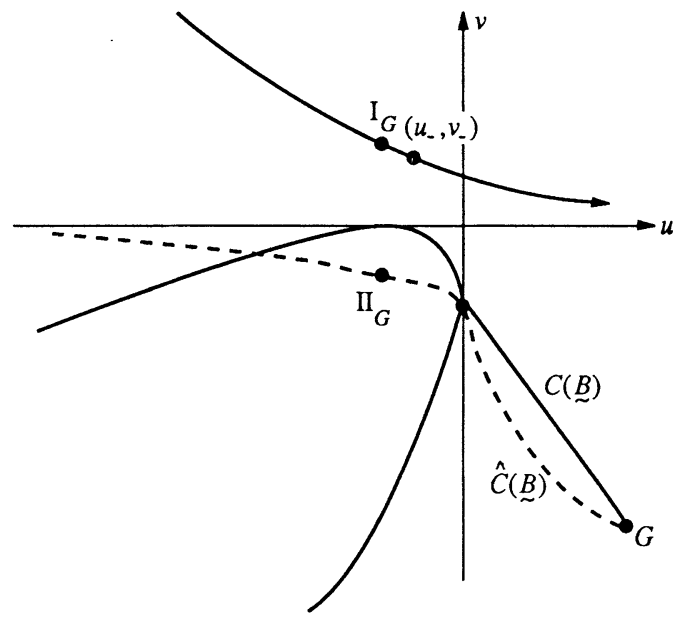

FIGURE 3.2

Due to (3.4), $u_{2} \rightarrow 0$ and $v_{2} \rightarrow-(a-1)$ as $u_{1} \rightarrow 0^{-}$, while $u_{2} \rightarrow-\infty$ and $v_{2} \rightarrow 0^{-}$as $u_{1} \rightarrow-(a-1)$. This implies, by (3.3), that ${\underset{\sim}{u_{1}} \rightarrow-(a-1)}^{v_{1}}$ and $u_{\underset{\sim}{B}} \rightarrow 0$ as $u_{1} \rightarrow 0$, while $v_{\underset{\sim}{B}} \rightarrow-\infty$ and $u_{\underset{\sim}{B}} \rightarrow \infty$ as $\underset{u_{1}}{u_{1}} \rightarrow-(a-1)$. Therefore, both the curves $C(\underset{\sim}{B})$ and $\widehat{C}(\underset{\sim}{B})$ start from the same state $Q$. Furthermore, the slope along the curve $\widehat{C}(\underset{\sim}{B})$ takes the form

$$
\frac{d v_{\stackrel{B}{~}}}{d u_{\underset{\sim}{B}}}=\frac{\sqrt{a-1}+\sqrt{-u_{2}}}{\frac{-1}{2}\left[\frac{\sqrt{a-1}}{\left(\sqrt{\left.-u_{2}+\sqrt{a-1}\right)^{2}}\right.} \cdot v_{2}+\sqrt{a-1}\right]+\frac{{\sqrt{-u_{2}}}^{2}}{\sqrt{-u_{2}+\sqrt{a-1}} \cdot \frac{d v_{2}}{d u_{2}}}}
$$

where $\left(u_{2}, v_{2}\right) \in C(\widetilde{B})$ with

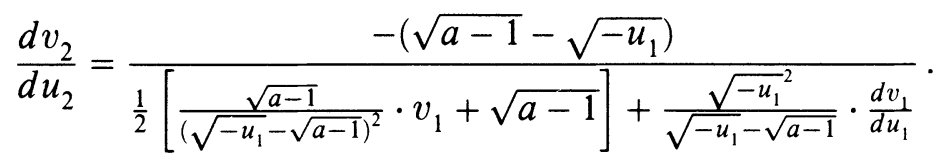

It is easy to see from (3.5) and (3.6) that the slope of the curve $\widehat{C}(\underset{\sim}{B})$ tends to negative infinite as $u_{1} \rightarrow 0^{-}$. This shows that the curve $C(\underset{\sim}{B})$ is located on the right-hand side of $\widehat{C}(\underset{\sim}{B})$ when $u_{1}<0$ and $\left|u_{1}\right|$ is small enough.

Now, suppose the curve $\widehat{C}(\underset{\sim}{B})$ intersects the curve $C(\underset{\sim}{B})$ at state $G$ the first time. Thus, there exist a state $\mathrm{I}_{G}$ on the curve $\bar{W}_{1}\left(u_{-}, v_{-}\right)$and a state $\mathrm{II}_{G}$ on the curve $C(\widetilde{B})$ respectively (see Figure 3.2$)$ such that the state $\left(u_{1}, v_{1}\right)$ on $\bar{W}_{1}\left(u_{-}, v_{-}\right)$corresponding to $G \in C\left(\underset{\sim}{\stackrel{B}{*})}\right.$ is $\mathrm{I}_{G}$ and the state $\left(u_{2}, v_{2}\right)$ on $C(\widetilde{B})$ corresponding to $G \in \widehat{C}(\underset{\sim}{B})$ is $\mathrm{II}_{G}$. Namely, the curve $S_{2}(G)$ passes 


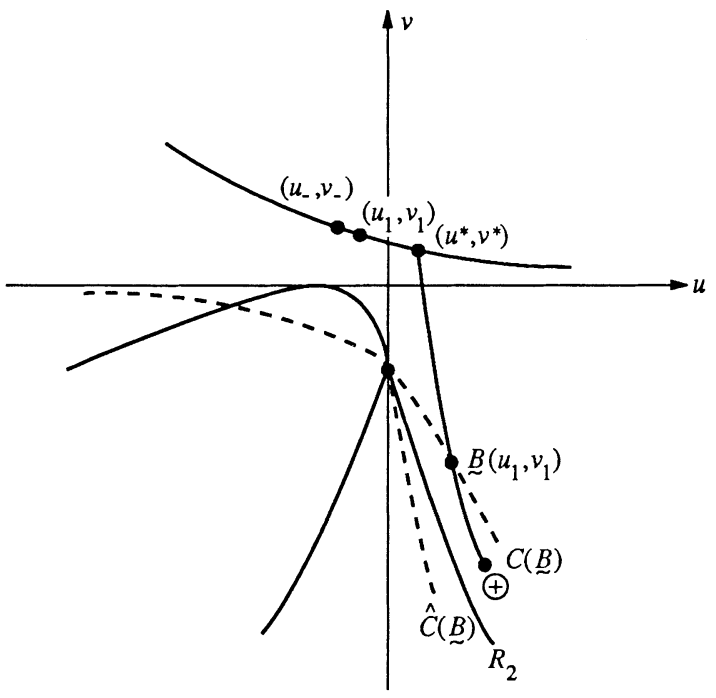

Figure 3.3

through both the states $\mathrm{II}_{G}$ and $\mathrm{I}_{G}$. But, this is impossible since $u_{\mathrm{I}_{G}}=u_{\mathrm{II}_{G}}$ while the slope of $S_{2}(G)$ is negative from $G$ to $\mathrm{I}_{G}$.

Therefore, the curve $C(\underset{\sim}{B})$ is located on the right-hand side of $\widehat{C}(\underset{\sim}{B})$ always and the Proposition 3.1 is proved.

For any $\left(u_{+}, v_{+}\right)$on the curves $K\left(u_{1}, v_{1}\right)$, there is a single-valued function $(u(\xi), v(\xi))\left(\xi=\frac{x}{t}\right)$ satisfying the items I-III in Definition 3.1 such that it contains three waves: the first kind wave joining $\left(u_{-}, v_{-}\right)$to $\left(u_{1}, v_{1}\right)$; the 2-shock joining $\left(u_{1}, v_{1}\right)$ to $\underset{\sim}{B}\left(u_{1}, v_{1}\right)$ and the 2-rarefaction wave or 2-shock joining $\underset{\sim}{B}\left(u_{1}, v_{1}\right)$ to $\left(u_{+}, v_{+}\right)$. Let us denote the above single-valued function $(u(\xi), v(\xi))$ containing three waves by $(u, v)_{k_{R}}$ or $(u, v)_{k_{s}}$, respectively.

Proposition 3.2. For any $\left(u_{+}, v_{+}\right)$on the curves $K\left(u_{1}, v_{1}\right)$, there exists a singlevalued function $(u(\xi), v(\xi))$ satisfying the items I-III in Definition 3.1 such that the sum of the strength of all of the jumps contained is smaller than the above $(u, v)_{k}$ 's.

Proof. Consider $(u, v)_{K_{R}}$ first for which $\left(u_{+}, v_{+}\right)$is on the curve labelled $R_{2}\left(\underset{\sim}{B}\left(u_{1}, v_{1}\right)\right)$. For definiteness, let us assume that $0>u_{1}>u_{-}$. By virtue of the characterization of rarefaction wave curves (Figure 2.2) there is a state $\left(u^{*}, v^{*}\right)$ on $\bar{W}_{1}\left(u_{-}, v_{-}\right)$such that $\left(u_{+}, v_{+}\right)$is on the curve $R_{2}\left(u^{*}, v^{*}\right)$ (Figure 3.3). We obtain a new single-valued function $(u(\xi), v(\xi))$ satisfying the items I-III in Definition 3.1 then which contains a 1-rarefaction wave joining $\left(u_{-}, v_{-}\right)$to $\left(u^{*}, v^{*}\right)$ and a 2-rarefaction wave joining $\left(u^{*}, v^{*}\right)$ to $\left(u_{+}, v_{+}\right)$. The sum of the strength of all the jumps now is zero which is less than the $(u, v)_{K_{R}}$ 's obviously. 


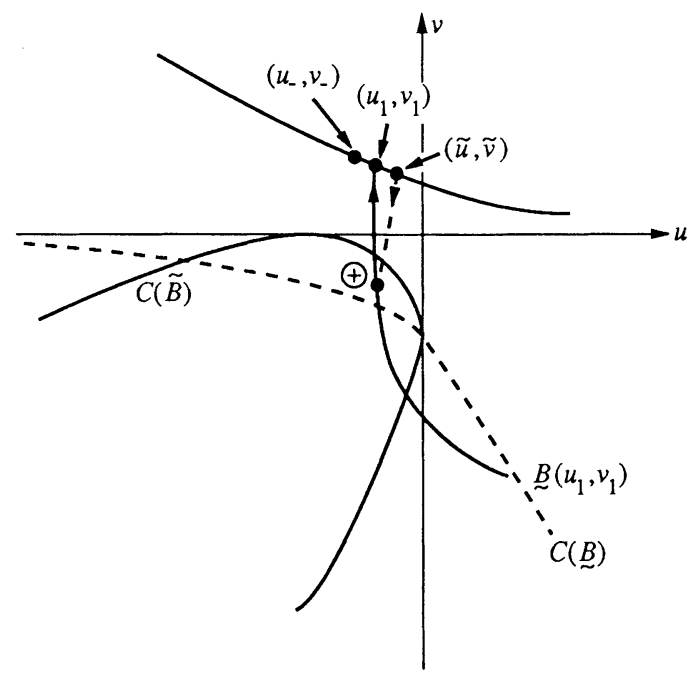

FIGURE 3.4

Turn to $(u, v)_{K_{S}}$ now for which there are different cases concerning the location of $\left(u_{+}, v_{+}\right)$. When $\left(u_{+}, v_{+}\right)$is on $S_{2}\left(\underset{\sim}{B}\left(u_{1}, v_{1}\right)\right)$ with $u_{+}>0$, similar to the above discussion, there exists a state $\left(u^{*}, v^{*}\right)$ on $\bar{W}_{1}\left(u_{-}, v_{-}\right)$with $u^{*}>0$ such that $\left(u_{+}, v_{+}\right)$is on the $R_{2}\left(u^{*}, v^{*}\right)$ and the corresponding sum of the strength of all the jumps is zero. When $\left(u_{+}, v_{+}\right)$is on $S_{2}\left(\underset{\sim}{B}\left(u_{1}, v_{1}\right)\right)$ with $u_{+}<0, v_{+}<0$, there are two possibilities corresponding to whether $\left(u_{+}, v_{+}\right)$is above or below the curve $C(\widetilde{B})$. For the former, there exists a state $(\tilde{u}, \tilde{v})$ on $\bar{W}_{1}\left(u_{-}, v_{-}\right)$with $u_{1}<\tilde{u}<0$ such that $\left(u_{+}, v_{+}\right)$is on the curve $S_{2}(\tilde{u}, \tilde{v})$ and the corresponding single-valued function $(u(\xi), v(\xi))$ contains a 1-rarefaction wave joining $\left(u_{-}, v_{-}\right)$to $(\tilde{u}, \tilde{v})$ (or a 1-shock but weaker than the one in $(u, v)_{K_{s}}$ 's) and a 2-shock joining $(\tilde{u}, \tilde{v})$ to $\left(u_{+}, v_{+}\right)$for which the sum of the strength of all jumps is much less than $(u, v)_{K_{S}}$ 's (see Figure 3.4). For the latter, it can be shown that there exist a point $(\bar{u}, \bar{v})$ on $\bar{W}_{1}\left(u_{-}, v_{-}\right)$ with $u_{1}<\bar{u}<0$ and the corresponding points $\widetilde{B}(\bar{u}, \bar{v})$ on the curve $C(\widetilde{B})$ and $\underset{\sim}{B}(\widetilde{B}(\bar{u}, \bar{v}))$ on the curve $\widehat{C}(\underset{\sim}{B})$ (see Figure 3.5) such that $\left(u_{+}, v_{+}\right)$is on the curve $S_{2}(\underset{\sim}{B}(\widetilde{B}(\bar{u}, \bar{v})))$ and the corresponding single-valued function $\left(u(\xi, v(\xi))\right.$ contains a first kind wave $\left(R_{1}\right.$ or $\left.S_{1}\right)$ joining $\left(u_{-}, v_{-}\right)$to $(\bar{u}, \bar{v})$; a 2-shock joining $(\bar{u}, \bar{v})$ to $\widetilde{B}(\bar{u}, \bar{v})$; a 2-shock joining $\widetilde{B}(\bar{u}, \bar{v})$ to $\underset{\sim}{B}(\widetilde{B}(\bar{u}, \bar{v}))$ and a 2-shock joining $\underset{\sim}{B}(\widetilde{B}(\bar{u}, \bar{v}))$ to $\left(u_{+}, v_{+}\right)$. Clearly, the sum of the strength of all of the jumps is less than $(u, v)_{K_{S}}$ 's again. The case when $\left(u_{+}, v_{+}\right)$is on the curve $S_{2}\left(\underset{\sim}{B}\left(u_{1}, v_{1}\right)\right)$ with $u_{+}<0, v_{+}>0$ is the same as the above former case.

For $u_{1} \leq u_{-}$, the discussion is similar.

Remark 3.3. For any fixed $\left(u_{1}, v_{1}\right) \in \bar{W}_{1}\left(u_{-}, v_{-}\right)$with $u_{1}<0$, draw $S_{2}\left(u_{1}, v_{1}\right) \quad\left(u \leq u_{1}\right)$. For any $\left(u_{2}, v_{2}\right) \in S_{2}\left(u_{1}, v_{1}\right)$ with $u_{2} \leq u_{1}, v_{2}>0$, 


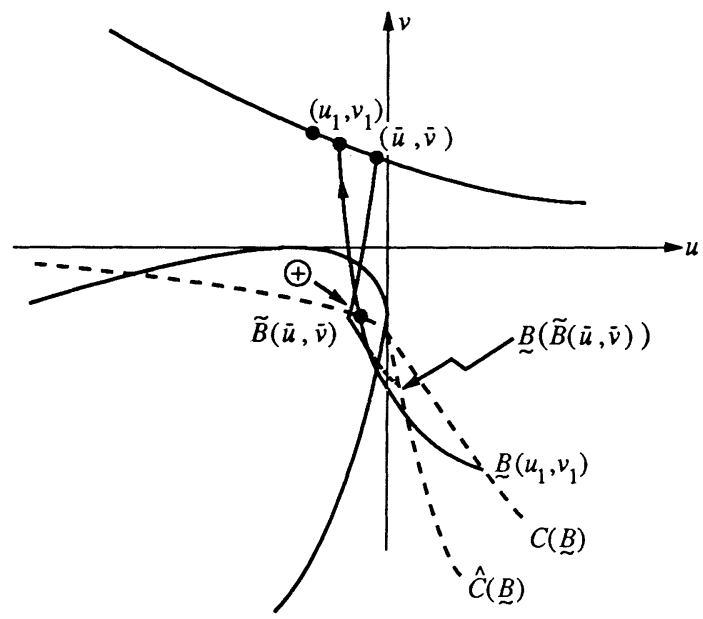

FIGURE 3.5

there exists the state $\underset{\sim}{B}\left(u_{2}, v_{2}\right)$ which can be joined to $\left(u_{1}, v_{1}\right)$, on the righthand side, by a single-valued function $(u(\xi), v(\xi))$ satisfying the items I-III in Definition 3.1. Namely,

$$
\left(u_{1}, v_{1}\right) \stackrel{s_{2}}{\rightarrow}\left(u_{2}, v_{2}\right) \stackrel{s_{2}}{\rightarrow} \underset{\sim}{B}\left(u_{2}, v_{2}\right) .
$$

We define the curve $\widehat{K}\left(u_{2}, v_{2}\right)$ which is $S_{2}\left(u_{1}, v_{1}\right)$ for $v_{2} \leq v<v_{1}$ and $S_{2}\left(\underset{\sim}{B}\left(u_{2}, v_{2}\right)\right)$ for $v_{2}>v \geq v_{\underset{\sim}{B}\left(u_{2}, v_{2}\right)}$, and $R_{2}\left(\underset{\sim}{B}\left(u_{2}, v_{2}\right)\right)$ for $v<v_{\underset{\sim}{B}\left(u_{2}, v_{2}\right)}$. This supplies a family of curves $\left\{\widehat{K}\left(u_{2}, v_{2}\right) ;\left(u_{2}, v_{2}\right) \in S_{2}\left(u_{1}, v_{1}\right), u_{2} \leq u_{1}\right.$, $v_{2}>0,\left(u_{1}, v_{1}\right)$ fixed $\}$ as $\left(u_{2}, v_{2}\right)$ varies along $S_{2}\left(u_{1}, v_{1}\right)\left(u<u_{1}\right)$ from $v_{2}=v_{1}$ to $v_{2} \rightarrow 0$ and this family of curves does belong to the set $\bar{W}_{2}\left(u_{1}, v_{1}\right)$, obviously. However, it can be shown, by the similar argument used in Remark 3.2 , that the family of curves $\{\widehat{K}\}$ violates item IV in Definition 3.1 and hence it does not belong to the family $\{A \bar{W}\}\left(u_{-}, v_{-}\right)$.

The whole plane $(u, v)$ is covered by the family $\{A \bar{W}\}\left(u_{-}, v_{-}\right)$univalently and is divided into ten subdomains as shown in Figure 3.6. For any state $\left(u_{+}, v_{+}\right) \in \mathrm{I}\left(u_{-}, v_{-}\right)$the solution consists of a 1-shock and a 2-rarefaction wave. For any state $\left(u_{+}, v_{+}\right) \subset \operatorname{II} \cup \operatorname{IV}\left(u_{-}, v_{-}\right)$, the solution consists of a 1rarefaction wave and a 2 -rarefaction wave. For any $\left(u_{+}, v_{+}\right) \in \operatorname{IIIUVI}\left(u_{-}, v_{-}\right)$, the solution consists of a 1-rarefaction wave and a 2-shock wave. For $\left(u_{+}, v_{+}\right) \in$ $V\left(u_{-}, v_{-}\right)$, the solution consists of a 1-shock wave and a 2-shock wave.

For $\left(u_{+}, v_{+}\right) \in \operatorname{VII}\left(u_{-}, v_{-}\right)$, confined by the curves $C(\widetilde{B}), S_{2}\left(\underset{\sim}{B}{ }^{-*}\right)$ and $\widehat{C}(\underset{\sim}{B})$ where $\underset{\sim}{B}{ }^{-*}=\underset{\sim}{B}\left(\widetilde{B}\left(u_{-}, v_{-}\right)\right)$, the wave pattern is $S_{1}-S_{2}-S_{2}-S_{2}$. More precisely, there exist states $(1) \in S_{1}\left(u_{-}, v_{-}\right)$and $(2) \in C(\widetilde{B}),(2)=$ $\widetilde{B}((1))$, and $(3) \in \widehat{C}(\underset{\sim}{B}),(3)=\underset{\sim}{B}((2))$, such that $(1)$ joins to $\left(u_{-}, v_{-}\right)$on the right by a 1 -shock with speed $\sigma_{1}((1) ;(-)) ;(2)$ joins to (1) on the right by a 2-shock with speed $\sigma_{2}((2) ;(1)) ;(3)$ joins to $(2)$ on the right by a 2-shock with 


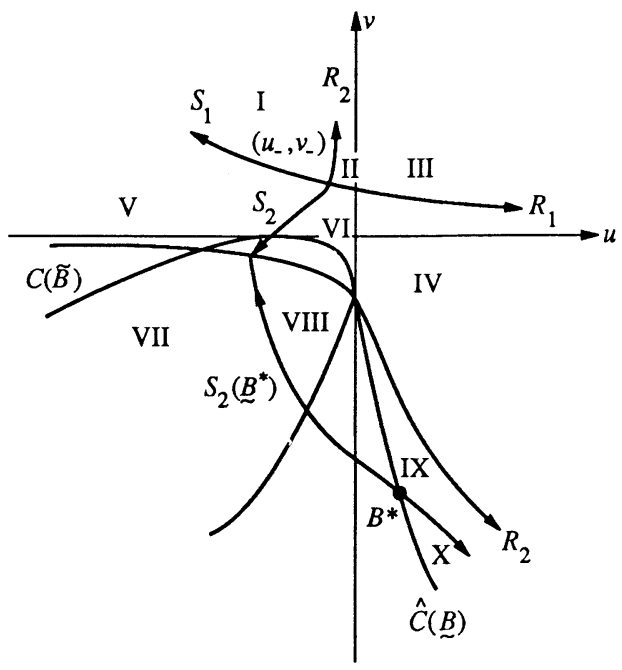

FIGURE 3.6

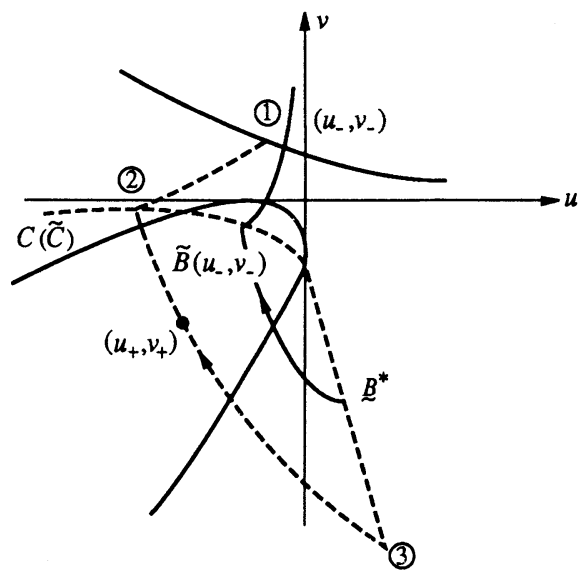

FIGURE 3.7

speed $\sigma_{2}((3) ;(2))$. Finally $\left(u_{+}, v_{+}\right) \in S_{2}((3))$ and joins to (3) on the right by a 2-shock with speed $\sigma_{2}((+) ;(3))$ (see Figure 3.7) where

$$
\begin{aligned}
\sigma((1) ;(-1)) & =\frac{1}{2}\left\{u_{-}-v_{-}+a+1-\sqrt{\left(v_{-}-u_{1}+(a-1)^{2}+4(a-1) u_{1}\right.}\right\} \\
\sigma_{2}((2) ;(1)) & =a-\sqrt{(a-1)\left(-u_{1}\right)}, \\
\sigma_{2}((3) ;(2)) & =a+\sqrt{(a-1)\left(-u_{2}\right)}, \\
\sigma_{2}((+) ;(3)) & =\frac{1}{2}\left\{u_{3}-v_{+}+a+1+\sqrt{\left(v_{+}-u_{3}+a-1\right)^{2}+4(a-1) u_{3}}\right\},
\end{aligned}
$$




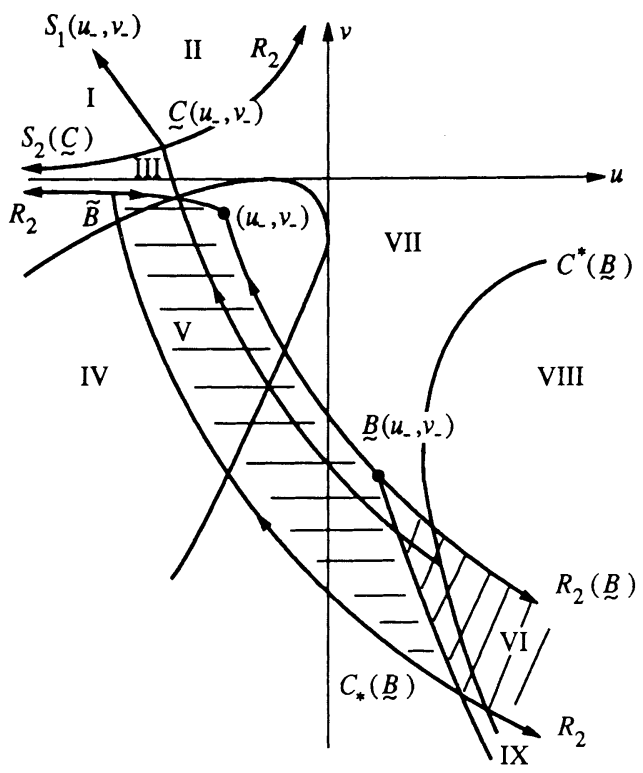

FIGURE 3.8

$$
\begin{aligned}
& \left\{\begin{array}{l}
u_{3}=u_{\sim_{((2))}}=\frac{\sqrt{-u_{2}}}{\sqrt{-u_{2}+\sqrt{a-1}}} v_{2}+\sqrt{(a-1)\left(-u_{2}\right)}, \\
v_{3}=v_{\mathcal{B}_{((2))}}=-\left(\sqrt{a-1}+\sqrt{-u_{2}}\right)^{2},
\end{array}\right. \\
& \left\{\begin{array}{l}
u_{2}=u_{\widetilde{B}((1))}=\frac{\sqrt{-u_{1}}}{\sqrt{-u_{1}-\sqrt{a-1}}} v_{1}-\sqrt{(a-1)\left(-u_{1}\right)}, \\
v_{2}=v_{\widetilde{B}((1))}=-\left(\sqrt{a-1}-\sqrt{-u_{1}}\right)^{2} .
\end{array}\right.
\end{aligned}
$$

It can be easily checked that $\sigma_{1}((1) ;(-))<\sigma_{2}((2) ;(1))<\sigma_{2}((3) ;(2))<$ $\sigma_{2}((+) ;(3))$ which shows that the sequence of 2-waves does not collapse into a single 2 -wave and we do have a single-valued function $(u(\xi)), v(\xi))$ with wave pattern $S_{1}-S_{2}-S_{2}-S_{2}$.

For $\left(u_{+}, v_{+}\right) \in \mathrm{IX}$, we have wave pattern $R_{1}-S_{2}-S_{2}-R_{2}$ and for $\left(u_{+}, v_{+}\right) \in \mathrm{X}$, we have $S_{1}-S_{2}-S_{2}-R_{2}$. In summary, there are eight different kinds of wave patterns: $R_{1}-R_{2} ; R_{1}-S_{2} ; S_{1}-R_{2} ; S_{1}-S_{2} ; R_{1}-S_{2}-S_{2}-S_{2}$; $R_{1}-S_{2}-S_{2}-R_{2} ; S_{1}-S_{2}-S_{2}-S_{2} ; S_{1}-S_{2}-S_{2}-R_{2}$.

Case 2. $\left(u_{-}, v_{-}\right) \in \mathrm{IV}$ in Figure 2.8 (namely $\left.\Delta\left(u_{-}, v_{-}\right)<0\right)$. For definiteness, assume $v_{-}>-(a-1),-(a-1)>u_{-}>-\sqrt{a-1}\left(\sqrt{a-1}+\sqrt{-v_{-}}\right)$for which case we have Figure 2.29. It can be shown that the whole plane $(u, v)$ is covered by the family $\{A \bar{W}\}\left(u_{-}, v_{-}\right)$univalently and divided into nine subdomains as follows. (See Figure 3.8 where the point $\underset{\sim}{C}, \widetilde{B}$ and $\underset{\sim}{B}$ are defined in Figure 2.29.)

Corresponding to each state $\left(u_{*}, v_{*}\right)$ on the curves $R_{2}(\widetilde{B})$ and $S_{2}(\widetilde{B})$ (which passes through the state $\left(u_{-}, v_{-}\right)$and stops there), the state $\underset{\sim}{B}\left(u_{*}, v_{*}\right)$ is defined (see the formulae (2.16), (2.18), replacing $\left(u_{*}, v_{*}\right)$ for $\left(u_{0}, v_{0}\right)$ there).

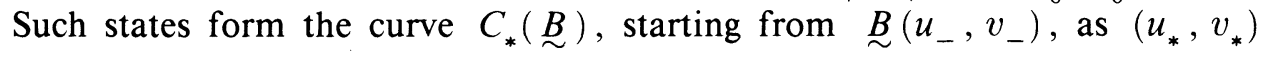




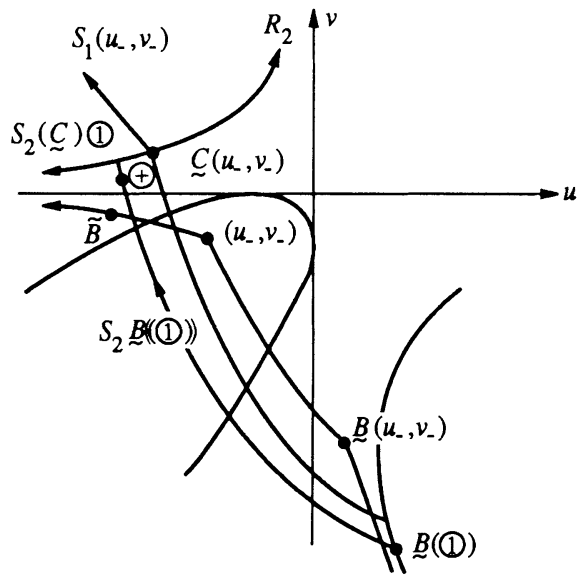

FIGURE 3.9

varies along $R_{2}(\widetilde{B}) \cup S_{2}(\widetilde{B})$ from $\left(u_{-}, v_{-}\right)$to $(u, v) \rightarrow\left(-\infty, 0^{-}\right)$. The curve $C_{*}(\underset{\sim}{B})$ tends to infinity: $u \rightarrow+\infty, v \rightarrow-\infty$. Corresponding to each state $\left(u^{*}, v^{*}\right)$ on the curve $S_{2}(\underset{\sim}{C})$ and $R_{2}(\underset{\sim}{C})$, the state $\underset{\sim}{B}\left(u^{*}, v^{*}\right)$ is defined (see the formula (2.16), (2.18), replacing $\left(u^{*}, v^{*}\right)$ for $\left(u_{0}, v_{0}\right)$ there). Such states form the curve $C^{*}(\underset{\sim}{B})$. It can be shown that $C^{*}(\underset{\sim}{B})$ does not intersect with $C_{*}(\underset{\sim}{B})$ and tends to infinity $(u \rightarrow \infty, v \rightarrow-\infty)$ as $u^{*} \rightarrow-\infty, v^{*} \rightarrow 0$; tends to

$$
\left\{\begin{array}{l}
v \rightarrow-(a-1) \\
u \rightarrow+\infty
\end{array}\right.
$$

as

$$
\left\{\begin{array}{l}
u^{*} \rightarrow 0 \\
v^{*} \rightarrow+\infty
\end{array}\right.
$$

For $\left(u_{+}, v_{+}\right) \in \mathrm{I}\left(u_{-}, v_{-}\right)$, the solution consists of a 1-shock, joining $\left(u_{-}\right.$, $\left.v_{-}\right)$to a state $(1) \in S_{1}\left(u_{-}, v_{-}\right)$, and a 2-shock, joining (1) and $\left(u_{+}, v_{+}\right)$. For $\left(u_{+}, v_{+}\right) \in \mathrm{II}\left(u_{-}, v_{-}\right)$, the solution consists of a 1-shock and a 2-rarefaction wave. For $\left(u_{+}, v_{+}\right) \in \operatorname{III}\left(u_{-}, v_{-}\right)$the solution consists of a 1-shock (joins $\left(u_{-}, v_{-}\right)$to $\underset{\sim}{C}$ ), a 2-shock (joins $\underset{\sim}{C}$ to a state $(1) \in S_{2}(\underset{\sim}{C})$ ), a 2-shock (joins (1) to $\underset{\sim}{B}(1) \in$ the curve $\left.C^{*}(\underset{\sim}{B})\right)$, a 2-shock joining $B((1))$ and $\left(u_{+}, v_{+}\right) \in$ $S_{2}(\underset{\sim}{B}(1))$ (see Figure 3.9).

For $\left(u_{+}, v_{+}\right) \in \operatorname{IV}\left(u_{-}, v_{-}\right)$, the solution consists of a 2-shock (which joins $\left(u_{-}, v_{-}\right)$to $\left.\widetilde{B}\left(u_{-}, v_{-}\right)\right)$, a 2-rarefaction wave (which joins $\widetilde{B}\left(u_{-}, v_{-}\right)$to a state $(1) \in R_{2}(\widetilde{B})$, a 2-shock (which joins (1) to $\underset{\sim}{B}(1) \in C_{*}(\underset{\sim}{B})$ ), a 2-shock (which joins $\underset{\sim}{B}(1)$ to $\left(u_{+}, v_{+}\right) \in S_{2}(\underset{\sim}{B}(1))$ (see Figure 3.10).

For $\left(u_{+}, v_{+}\right) \in \mathrm{V}\left(u_{-}, v_{-}\right)$, the wave pattern is $S_{2}-S_{2}-S_{2}-S_{2}$ (see Figure 3.11).

For $\left(u_{+}, v_{+}\right) \in \operatorname{VII}\left(u_{+}, v_{+}\right)$, the wave pattern is $S_{1}-R_{2}-S_{2}-S_{2}$ (Figure 3.12). 


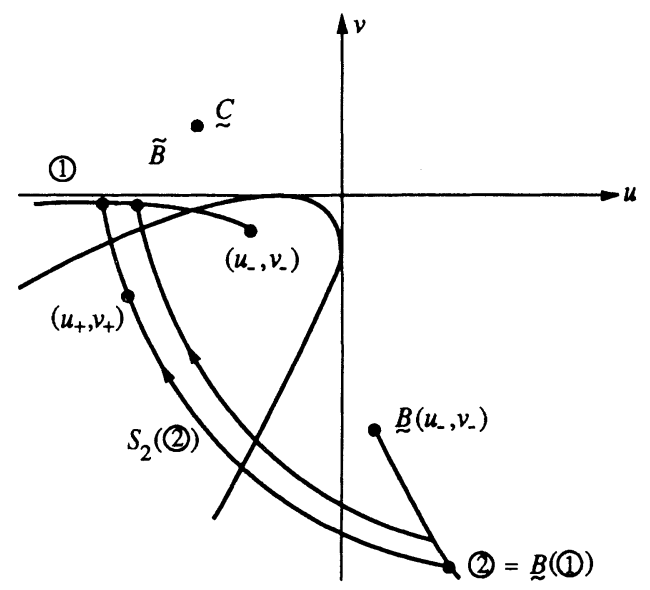

FIGURE 3.10

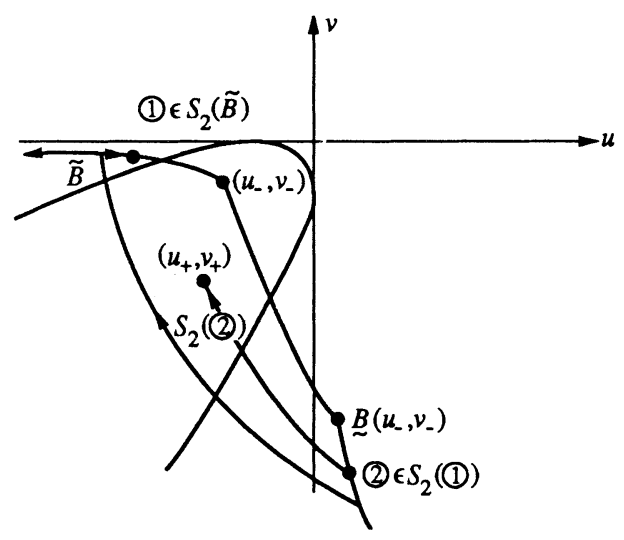

FIGURE 3.11. $\left(u_{-}, v_{-}\right) \stackrel{S_{2}}{\rightarrow} \widetilde{B} \stackrel{S_{2}}{\rightarrow}(1) \stackrel{S_{2}}{\rightarrow}(2) \stackrel{S_{2}}{\rightarrow}\left(u_{+}, v_{+}\right)$

For $\left(u_{+}, v_{+}\right) \in \operatorname{VIII}\left(u_{-}, v_{-}\right)$, the wave pattern is $S_{1}-R_{2}-S_{2}-R_{2}$ (Figure 3.13).

For $\left(u_{+}, v_{+}\right) \in \operatorname{VI}\left(u_{-}, v_{-}\right)$, the wave pattern is $S_{2}-S_{2}-S_{2}-R_{2}$.

For $\left(u_{+}, v_{+}\right) \in \operatorname{IX}\left(u_{-}, v_{-}\right)$, the wave pattern is $S_{2}-R_{2}-S_{2}-R_{2}$.

In summary, there are nine different kinds of wave pattern in these two cases.

$$
\begin{aligned}
& S_{1}-S_{2} ; \quad S_{1}-R_{2} ; \quad S_{2}-S_{2}-S_{2}-R_{2} ; \quad S_{2}-R_{2}-S_{2}-R_{2} ; \\
& S_{1}-S_{2}-S_{2}-S_{2} ; \quad S_{1}-R_{2}-S_{2}-S_{2} ; \quad S_{1}-R_{2}-S_{2}-R_{2} ; \\
& S_{2}-R_{2}-S_{2}-S_{2} ; \quad S_{2}-S_{2}-S_{2}-S_{2} \text {. }
\end{aligned}
$$

The other cases can be discussed by a similar argument. The most complicated structure of the solution consists of four waves joining by five states respectively. Moreover, it may happen that all of the four waves belong to the second family. 


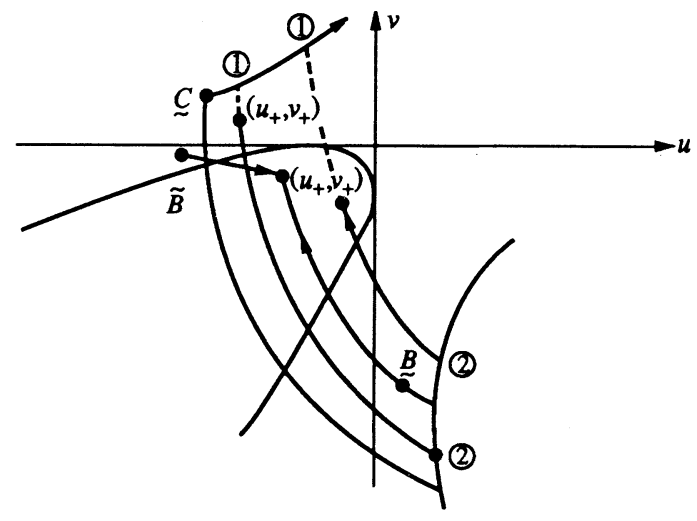

FIGURE 3.12. $\left(u_{-}, v_{-}\right) \stackrel{S_{1}}{\rightarrow} \underset{\sim}{\stackrel{R_{2}}{\rightarrow}}(1) \stackrel{S_{2}}{\rightarrow}(2) \stackrel{S_{2}}{\rightarrow}\left(u_{+}, v_{+}\right)$, there are different location of $\left(u_{+}, v_{+}\right)$

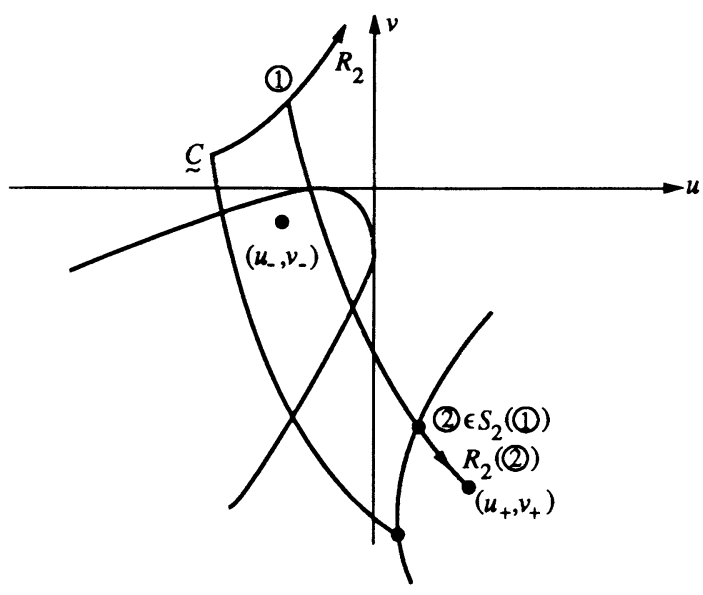

FIGURE 3.13. $\left(u_{-}, v_{-}\right) \stackrel{S_{1}}{\rightarrow} \underset{\sim}{\stackrel{R_{2}}{\rightarrow}}(1) \stackrel{S_{2}}{\rightarrow}(2) \stackrel{R_{2}}{\rightarrow}\left(u_{+}, v_{+}\right)$

We have shown that for any given $\left(u_{-}, v_{-}\right)$the family $\{A \bar{W}\}\left(u_{-}, v_{-}\right)$can be defined, covering the whole plane $(u, v)$ univalently. The above results can be summarized as follows

Theorem 3.1. For any given $\left(u_{-}, v_{-}\right)$and $\left(u_{+}, v_{+}\right)$, there exists a unique admissible weak solution of the Riemann problem (1.1) and (1.3).

\section{REFERENCES}

[BN] J. H. Bick and G. F. Newell, A continuum model for two-directional traffic flow, Quart. Appl. Math. 18 (1961), 191-204.

[Ha] H. Hattori, The Riemann problem for a Van der Waals fluid with entropy rate admissibility criterion-Isothermal case, Arch. Rational Mech. Anal. (to appear). 
[Ho] $\mathrm{H}$. Holden, On the Riemann problem for a prototype of a mixed type conservation law, Comm. Pure. Appl. Math. 40 (1987), 229-264.

$\left[\mathrm{Hs}_{1}\right] \quad \mathrm{L}$. Hsiao, Admissible weak solution for nonlinear system of conservation laws in mixed type J. Partial Differential Equations 21 (1989), 40-58.

$\left[\mathrm{HS}_{2}\right] \ldots$, Qualitative behavior of solutions for Riemann problems of conservation laws of mixed type, Proc. of the Second International Conference on Nonlinear Hyperbolic Problems, Aachen, FRG, March 14-18, 1988, Notes on Numerical Fluid Mechanics, Vol. 24, 1989, pp. 246-256.

[H-M] L. Hasiao and P. de Mottoni, Quasilinear hyperbolic system of conservation laws with parabolic degeneracy, Rocky Mountain J. Math. (to appear).

[J] R. D. James, The propagation of phase boundaries in elastic bars, Arch. Rational Mech. Anal. 73 (1980), 125-158.

[K] B. L. Keyfitz, The Riemann problem for nonmonotone stress-strain functions: A "Hysteresis" approach (to appear).

[L] T. P. Liu, The Riemann problem for general $2 \times 2$ conservation laws, Trans. Amer. Math. Soc. 199 (1974), 89-112.

[M-C] J. D. Murray and J. E. R. Cohen, On nonlinear convective dispersal effects in an interacting population model, SIAM. J. Appl. Math. 43 (1983), 66-78.

[Se] M. Shearer, The Riemann problem for a class of conservation laws of mixed type, J. Differential Equations 46 (1982), 426-443.

[Sl] M. Slemrod, Admissibility criteria for propagating phase boundaries in a Van der Waals fluid, Arch. Rational Mech. Anal. 81 (1983), 301-315.

[SS] D. G. Schaeffer and M. Shearer, Riemann problem for nonstrictly hyperbolic $2 \times 2$ systems of conservation laws, Trans. Amer. Math. Soc. 304 (1987), 267-306.

[T] B. Temple, Global solutions of the Cauchy problem for a class of $2 \times 2$ nonstrictly hyperbolic conservation laws, Adv. Appl. Math. 3 (1982), 335-375.

Academia Sinica, Institute of Mathematics, Beijing, China

Dipartimento di Matematica, II Università Degli Studi di Roma, Roma I-00173, Italy 